ESAIM: COCV 18 (2012) 1027-1048

DOI: $10.1051 / \mathrm{cocv} / 2011193$
ESAIM: Control, Optimisation and Calculus of Variations

www.esaim-cocv.org

\title{
AN ANALYSIS OF ELECTRICAL IMPEDANCE TOMOGRAPHY WITH APPLICATIONS TO TIKHONOV REGULARIZATION
}

\author{
Bangti Jin ${ }^{1}$ And Peter MaAss ${ }^{2}$
}

\begin{abstract}
This paper analyzes the continuum model/complete electrode model in the electrical impedance tomography inverse problem of determining the conductivity parameter from boundary measurements. The continuity and differentiability of the forward operator with respect to the conductivity parameter in $L_{p}$-norms are proved. These analytical results are applied to several popular regularization formulations, which incorporate a priori information of smoothness/sparsity on the inhomogeneity through Tikhonov regularization, for both linearized and nonlinear models. Some important properties, e.g., existence, stability, consistency and convergence rates, are established. This provides some theoretical justifications of their practical usage.
\end{abstract}

Mathematics Subject Classification. 49N45, 65N21.

Received December 23, 2010. Revised June 1st, 2011.

Published online 16 January 2012.

\section{INTRODUCTION}

Electrical impedance tomography (EIT) is an emerging imaging modality, and it has attracted much interest in noninvasive imaging and nondestructive testing. For instance, the reconstructions can be used for diagnostic purposes in medical applications, e.g., monitoring of lung function, detection of cancer in the skin and breast and location of epileptic foci [4]. A similar inverse problem arises in geophysics, where one uses electrodes on the surface of the earth or in bore holes to locate resistivity anomalies, e.g., minerals or contaminated sites.

A typical experimental setup is as follows. One first applies an electrical current through the electrodes attached to the surface of the object, and then measures the resulting electrical potential on the electrodes. The procedure is repeated several times with different input currents, which yields a partial information about the Neumann-to-Dirichlet map (or its discrete approximation). EIT aims at determining a spatially-varying physical electrical conductivity of the object by using these noisy measurements.

This inverse problem was first formulated by Calderón [9], who also gave a first uniqueness result for the linearized problem. The mathematical theory of unique solvability of the inverse problem with the complete Neumann-to-Dirichlet map has received much attention, and many profound theoretical results have been obtained. For a comprehensive overview of uniqueness results, we refer to the survey [55], also [2], where in

\footnotetext{
Keywords and phrases. Electrical impedance tomography, Tikhonov regularization, convergence rate.

1 Department of Mathematics and Institute for Applied Mathematics and Computational Sciences, Texas A\&M University, College Station, 77843-3368 TX, USA. btjin@math.tamu.edu

2 Center for Industrial Mathematics, University of Bremen, 28334 Bremen, Germany. pmaass@math.uni-bremen.de
} 
dimension two, the uniqueness in the class of $L_{\infty}(\Omega)$ functions was shown, and [31] for partial data. Stability issues of the inverse problem have also been extensively investigated [1].

The broad prospective applications have aroused much interest in designing efficient imaging algorithms. A large number of numerical methods have been proposed in the literature, and roughly they can be divided into two categories. The former relies on refined analytical properties of the forward model, whereas the latter applies general-purposed regularization strategies. Imaging algorithms in the first category utilize powerful analytical machineries, e.g., spectral analysis and complex geometrical optics, and include factorization method [39], enclosure method [30] and d-bar method [32,40] etc. These methods can be very effective for specific situations, e.g., estimating inclusion supports. Among methods of the latter category, the variational idea stands out prominently. It is based on minimizing a certain functional, typically the squared $L_{2}$ norm of the difference between the simulated boundary electrical potential due to an assumed conductivity and the measured potential. One standard approach of this type is described in [10], which applies one step of a Newton method with a constant conductivity as the initial guess, see also [43]. Some sort of regularization is beneficial to combat the numerical instability and to fully exploit a priori knowledge for achieving enhanced resolution [20,54]. As such, imaging algorithms based on Tikhonov regularization are very popular, and often taken as a benchmark. In [47], the standard Tikhonov regularization for EIT in unbounded domains was studied. In [52], the Mumford-Shah model was suggested in the hope of simultaneously segmenting the conductivity image and enhancing the resolution. In [33], a level set approach was developed for estimating the interface, and in [13], it was used for total variation $(T V)$ regularized $L_{2}$ data fitting to reconstruct a piecewise constant conductivity. Recently, the so-called sparsity penalty ( $c f$. Sect. 4 for details) was adopted to reconstruct conductivity distributions that have a sparse representation in a given basis/over-complete frame [36]. Simulation studies [36] and experimental evaluations [23] clearly show the great potentials of the sparsity ideas in EIT imaging. Iterative regularization methods, such as Landweber iteration and Gauss-Newton method, provide an alternative framework [20], see [43,45] for their applications in EIT. Also we refer to [37,41] for statistical inversion.

Although there are numerous numerical studies of these imaging algorithms in the literature, theoretical justifications of their excellent empirical performances remain scarce. Ikehata and Siltanen [30] investigated an extracting formula for locating inclusions from a finite number of EIT measurements, and established its convergence as the noise level tends to zero. Lechleiter [42] established the validity of the factorization method, regularized by spectral cutoff, for finding inclusions from finite-dimensional data. Knudsen et al. [40] analyzed a regularized variant of the d-bar method, and derived a convergence rate of logarithmic type in suitable Banach spaces for $C^{2}$ conductivities. As to more "standard" imaging algorithms based on Tikhonov regularization, their mathematical properties and convergence behaviors of related algorithms also have not received due attention despite their wide-spread practical adoption, and these procedures are largely applied in an ad hoc manner. Theoretically, we are only aware of $[16,47,51,52]$. In [16], a regularization approach based on wavelet was analyzed, especially the convergence of an iterative algorithm was established. However, the analysis allows only continuously differentiable conductivities. In [47], the consistency, stability and convergence rates were discussed under a high Sobolev regularity on the conductivity, which can possibly make its numerical realization inconvenient. In the interesting works [51,52], the existence and stability were established for Mumford-Shah and $T V$ penalties, respectively, and also consistency [51]. Therefore, the issue of convergence rate remains unexplored, and the popular $H^{1}$-smoothness penalty has not been addressed so far.

In this paper, we attempt to provide partial theoretical justifications of the methods based on Tikhonov regularization, i.e., with smoothness/sparsity penalty, for linearized and nonlinear models, especially convergence rates, along the line of $[51,52]$. We first develop necessary analytical machineries, including continuity and Fréchet differentiability of the forward operator in $L_{p}$-norms. This is achieved with the help of Meyers' celebrated gradient estimate $[22,25,48]$. Then we capitalize on recent theoretical developments $[8,24,28,34,35,46]$ for nonsmooth regularization to derive well-posedness and convergence rate results. Finally, when completing the manuscript, we got to know the interesting work [18], which analyzes the standard Tikhonov regularization for diffuse optical tomography. Although the underlying ideas of [18] and the present work are similar, there are some significant differences in the forward model as well as the formulations: The optical tomography forward 
model in [18] has a Robin boundary condition and their focus is on the standard $H^{1}$-penalty, while the EIT model has a Neumann-type boundary condition and we are interested in smoothness/sparsity penalty.

The rest of the paper is organized as follows. We develop necessary analytical results, including continuity and differentiability of the forward operator with respect to $L_{p}$ norms, for the continuum model in Section 2, which improve known results in the $L_{\infty}$ norm, and enable us to apply regularization theory in a Hilbert space, see Section 4. In Section 3 the extension to the practically popular complete electrode model $[11,53]$ is discussed. Then in Section 4, we describe regularization models with $\ell_{r}$ penalties, and study their properties, e.g., existence, stability, consistency and convergence rate, under various conditions. The conventional smoothness penalty is covered as a special case. We conclude in Section 5 with a brief discussion on related issues.

\section{Continuum MODEL}

This section studies the basic mathematical model, the continuum model, of the EIT problem. The main part is devoted to proving analytic properties of the parameter-to-state map and to establishing differentiability of the forward operator with respect to $L_{p}$ norms.

\subsection{Notation and definitions}

Let $\Omega$ be an open bounded domain in $\mathbb{R}^{d}(d=2,3)$ with a Lipschitz boundary $\Gamma$. Throughout this paper, we shall make use of the space $\tilde{H}^{1}(\Omega)$, which is a subspace of the Sobolev space $H^{1}(\Omega)$ with vanishing mean on the boundary $\Gamma$, i.e., $\tilde{H}^{1}(\Omega)=\left\{v \in H^{1}(\Omega): \int_{\Gamma} v \mathrm{~d} s=0\right\}$. The spaces $\tilde{H}^{\frac{1}{2}}(\Gamma)$ and $\tilde{H}^{-\frac{1}{2}}(\Gamma)$ are defined similarly. These spaces are equipped with the usual norms.

In the absence of interior current source and in the electrostatic state, Maxwell's system describing electromagnetic fields inside the object reduces to the following second-order elliptic partial differential equation

$$
-\nabla \cdot(\sigma \nabla u)=0 \text { in } \Omega
$$

with a Neumann boundary condition $\sigma \frac{\partial u}{\partial n}=j \in \tilde{H}^{-\frac{1}{2}}(\Gamma)$ on the boundary $\Gamma$. We normalize the solution by enforcing $\int_{\Gamma} u \mathrm{~d} s=0$ to ensure a unique solution $u \in \tilde{H}^{1}(\Omega)$, and denote by $F(\sigma)$ the forward operator.

In an EIT experiment, one measures a noisy version $\phi^{\delta} \in L_{2}(\Gamma)$ of the potential $\phi^{\dagger}=F\left(\sigma^{\dagger}\right) j\left(\sigma^{\dagger}\right.$ refers to the exact conductivity), with its accuracy measured by the noise level $\delta=\left\|\phi^{\dagger}-\phi^{\delta}\right\|_{L_{2}(\Gamma)}$. For a given pair $\left(j, \phi^{\delta}\right)$, the variational approach seeks an approximation to $\sigma^{\dagger}$ by minimizing a certain discrepancy functional together with an appropriate penalty over the following admissible set

$$
\mathcal{A}=\left\{\sigma \in L_{\infty}(\Omega): \lambda \leq \sigma \leq \lambda^{-1} \text { a.e. and } \operatorname{supp}\left(\sigma-\sigma^{\dagger}\right) \subset \Omega^{\prime}\right\},
$$

for some fixed $\lambda \in(0,1)$, where $\Omega^{\prime}$ is an open subset with a smooth boundary compactly contained in $\Omega$. We shall endow the set $\mathcal{A}$ with an $L_{p}(\Omega)$ norm $(1 \leq p \leq \infty)$.

Remark 2.1. The admissible set $\mathcal{A}$ is closed and convex, but it has no interior points, i.e., for any $\sigma \in \mathcal{A}$, the ball $B_{\epsilon}=\left\{\tilde{\sigma}:\|\tilde{\sigma}-\sigma\|_{L_{p}(\Omega)} \leq \epsilon\right\}$ is not completely contained in $\mathcal{A}$ for any $\epsilon>0$. Therefore, all the results presented below should be understood with respect to the relative topology.

In what follows $C$ denotes some generic constant, which may differ at different occurrences. We first state a well-known regularity estimate.

Lemma 2.2. For any $\sigma \in \mathcal{A}$, the forward solution $F(\sigma) j$ satisfies

$$
\|F(\sigma) j\|_{H^{1}(\Omega)} \leq C\|j\|_{H^{-\frac{1}{2}}(\Gamma)} .
$$

Proof. The estimate follows directly from Lax-Milgram theorem. 
Lemma 2.2 implies that the forward operator $F(\sigma)$ is uniformly bounded for a fixed $j$. We also recall the following norm equivalence result.

Lemma 2.3. On the space $\tilde{H}^{1}(\Omega)$, the standard $H^{1}(\Omega)$ norm is equivalent to the $H^{1}(\Omega)$ semi-norm, i.e., there exist two constants $c_{0}$ and $c_{1}$ such that for any $v \in \tilde{H}^{1}(\Omega)$

$$
c_{0}\|v\|_{H^{1}(\Omega)} \leq\|\nabla v\|_{L_{2}(\Omega)} \leq c_{1}\|v\|_{H^{1}(\Omega)} .
$$

For simplicity, we describe in this section only (continuity and differentiability) results for one fixed input current $j$, and suppress the dependence of the solution $u=F(\sigma) j$ on $j$ hereon. The extension to multiple data set, i.e., $\left\{\left(j_{i}, \phi_{i}\right)\right\}_{i=1}^{N}$ as often in practical scenarios or the full Neumann-to-Dirichlet map, is straightforward. We remind that, in case of Neumann-to-Dirichlet map, the norm in the discrepancy measure should be understood as the operator norm, see, e.g., [51,52].

\subsection{Continuity and differentiability}

The differentiability of the forward operator $F(\sigma)$ with respect to the $L_{\infty}$-norm is well-known. For instance, it was already noted in the pioneering work [9] that the forward map is analytic in $\sigma$ with respect to the $L_{\infty}$-norm. In [15], the Frechét differentiability was proved, and a general approach for trilinear forms (or with bilinear structure) was provided in [44]. However, these results are concerned with $L_{\infty}$ differentiability, which is insufficient for analyzing some Tikhonov functionals, including conventional $H^{1}$-smoothness/sparsity penalty.

We will derive a differentiability result in $L_{p}$ norms, which is crucial for analyzing the regularization formulations in Section 4. We shall prove continuity and Fréchet differentiability by applying Meyers' celebrated gradient estimates [48]. The proof techniques in this part are inspired by and closely follow [52]. We start by stating Meyers' gradient estimate [48] as formulated in [52].

Theorem 2.4. Let $\Omega$ be a bounded Lipschitz domain in $\mathbb{R}^{d}(d \geq 2)$. Assume that $\sigma \in L_{\infty}(\Omega)$ satisfies $\lambda<\sigma<$ $\lambda^{-1}$ for some fixed $\lambda \in(0,1)$. For $f \in\left(L_{q}(\Omega)\right)^{d}$ and $h \in L_{q}(\Omega)$, let $u \in H^{1}(\Omega)$ be a weak solution of

$$
-\nabla \cdot(\sigma \nabla u)=-\nabla \cdot f+h \text { in } \Omega .
$$

Then, there exists a constant $Q \in(2, \infty)$ depending on $\lambda$ and $d$ only, $Q \rightarrow 2$ as $\lambda \rightarrow 0$ and $Q \rightarrow \infty$ as $\lambda \rightarrow 1$, such that for any $2<q<Q$ we obtain $u \in W_{\text {loc }}^{1, q}(\Omega)$ and for any $\Omega_{1} \subset \subset \Omega$

$$
\|u\|_{W^{1, q}\left(\Omega_{1}\right)} \leq C\left(\|u\|_{H^{1}(\Omega)}+\|f\|_{L_{q}(\Omega)}+\|h\|_{L_{q}(\Omega)}\right),
$$

where the constant $C$ depends on $\lambda, d, q, \Omega_{1}$ and $\Omega$.

In this theorem the boundary condition for the differential equation can be general. Its effect enters the $W^{1, q_{-}}$ estimate through the term $\|u\|_{H^{1}(\Omega)}$. Otherwise, no further regularity has been assumed on the conductivity $\sigma$. In general, a precise estimate of the constant $Q(\lambda, d)$ is missing, although in the two-dimensional case, a sharp estimate of $Q(\lambda, d)$ was derived in [3]. We shall denote by $Q(\lambda)$ the number defined in Theorem 2.4 by suppressing its dependence on $d$.

Assisted with Theorem 2.4 and by repeatedly applying generalized Hölder's inequality, we show the continuity of the operator $F(\sigma)$ with respect to the $L_{p}$ norm for any $p \in\left(\frac{2 Q(\lambda)}{Q(\lambda)-2}, \infty\right]$.

Lemma 2.5. For the operator $F(\sigma)$ and $\sigma, \sigma+\vartheta \in \mathcal{A}$, we have the following continuity properties.

(a) For any $p \in\left(\frac{2 Q(\lambda)}{Q(\lambda)-2}, \infty\right]$ and $\sigma, \sigma+\vartheta \in \mathcal{A}$ we have

$$
\|F(\sigma+\vartheta)-F(\sigma)\|_{H^{1}(\Omega)} \leq C\|\vartheta\|_{L_{p}\left(\Omega^{\prime}\right)}
$$


(b) for any $p \in\left(\frac{4 Q(\lambda)}{Q(\lambda)-2}, \infty\right]$, there exists $q \in(2, Q(\lambda))$ such that

$$
\|F(\sigma+\vartheta)-F(\sigma)\|_{W^{1, q}\left(\Omega^{\prime}\right)} \leq C\|\vartheta\|_{L_{p}\left(\Omega^{\prime}\right)} ;
$$

(c) for $p \geq 1$ and any $q \in(2, Q(\lambda))$ we have the following estimates

$$
\lim _{\|\vartheta\|_{L_{p}\left(\Omega^{\prime}\right)} \rightarrow 0}\|F(\sigma+\vartheta)-F(\sigma)\|_{W^{1, q}\left(\Omega^{\prime}\right)}=0 .
$$

Proof. For $\sigma, \sigma+\vartheta \in \mathcal{A}$, the weak formulations of $F(\sigma)$ and $F(\sigma+\vartheta)$ give

$$
\int_{\Omega} \sigma \nabla F(\sigma) \cdot \nabla v \mathrm{~d} x=\int_{\Omega}(\sigma+\vartheta) \nabla F(\sigma+\vartheta) \cdot \nabla v \mathrm{~d} x, \quad \forall v \in \tilde{H}^{1}(\Omega),
$$

i.e.,

$$
\int_{\Omega} \sigma \nabla(F(\sigma)-F(\sigma+\vartheta)) \cdot \nabla v \mathrm{~d} x=\int_{\Omega} \vartheta \nabla F(\sigma+\vartheta) \cdot \nabla v \mathrm{~d} x, \quad \forall v \in \tilde{H}^{1}(\Omega) .
$$

Taking $v=F(\sigma)-F(\sigma+\vartheta) \in \tilde{H}^{1}(\Omega)$ in the equation and noting that the support of $\vartheta$ is within $\Omega^{\prime}$ give

$$
\begin{aligned}
\int_{\Omega} \sigma|\nabla(F(\sigma)-F(\sigma+\vartheta))|^{2} \mathrm{~d} x & =\int_{\Omega} \vartheta \nabla F(\sigma+\vartheta) \cdot \nabla(F(\sigma)-F(\sigma+\vartheta)) \mathrm{d} x \\
& =\int_{\Omega^{\prime}} \vartheta \nabla F(\sigma+\vartheta) \cdot \nabla(F(\sigma)-F(\sigma+\vartheta)) \mathrm{d} x \\
& \leq\|\vartheta\|_{L_{p}\left(\Omega^{\prime}\right)}\|\nabla F(\sigma+\vartheta)\|_{L_{q}\left(\Omega^{\prime}\right)}\|\nabla(F(\sigma)-F(\sigma+\vartheta))\|_{L_{2}(\Omega)},
\end{aligned}
$$

where $\frac{1}{p}+\frac{1}{q}=\frac{1}{2}$. The assumption $p \in\left(\frac{2 Q(\lambda)}{Q(\lambda)-2}, \infty\right]$ implies $q<Q(\lambda)$. By Theorem 2.4, there exists a constant $C$ such that

$$
\|\nabla F(\sigma+\vartheta)\|_{L_{q}\left(\Omega^{\prime}\right)} \leq C\|F(\sigma+\vartheta)\|_{H^{1}(\Omega)} \leq C\|j\|_{H^{-\frac{1}{2}}(\Gamma)} .
$$

This together with Lemma 2.3 shows

$$
\|F(\sigma)-F(\sigma+\vartheta)\|_{H^{1}(\Omega)} \leq C\|\vartheta\|_{L_{p}\left(\Omega^{\prime}\right)} .
$$

This shows the first part of the lemma.

To prove the second part, we fix $q \in(2, Q(\lambda))$ and choose $p=\frac{2 q}{q-2}$, i.e., $\frac{1}{p}+\frac{1}{q}=\frac{1}{2}$, and $p \in\left(\frac{4 Q(\lambda)}{Q(\lambda)-2}, \infty\right]$. We apply Meyers' theorem and obtain

$$
\|F(\sigma)-F(\sigma+\vartheta)\|_{W^{1, q}\left(\Omega^{\prime}\right)} \leq C\left(\|F(\sigma)-F(\sigma+\vartheta)\|_{H^{1}(\Omega)}+\|\vartheta \nabla F(\sigma+\vartheta)\|_{L_{q}(\Omega)}\right) .
$$

The first term in the bracket has been estimated in part $(a)$, and thus we only need to bound the term $\|\vartheta \nabla F(\sigma+\vartheta)\|_{L_{q}(\Omega)}$. Take any small $\epsilon$ such that $q^{\prime}=q+\epsilon \in(q, Q(\lambda))$ and $\frac{q q^{\prime}}{q^{\prime}-q} \geq p$. By appealing to Hölder's inequality, we deduce

$$
\begin{aligned}
\int_{\Omega}|\vartheta \nabla F(\sigma+\vartheta)|^{q} \mathrm{~d} x & =\int_{\Omega^{\prime}}|\vartheta|^{q}|\nabla F(\sigma+\vartheta)|^{q} \mathrm{~d} x \\
& \leq\left(\int_{\Omega^{\prime}}|\vartheta|^{\frac{q q^{\prime}}{q^{-q}}} \mathrm{~d} x\right)^{1-\frac{q}{q^{\prime}}}\left(\int_{\Omega^{\prime}}|\nabla F(\sigma+\vartheta)|^{q^{\prime}} \mathrm{d} x\right)^{\frac{q}{q^{\prime}}} \\
& \leq C\|j\|_{H^{-\frac{1}{2}}(\Omega)}^{q}\left(\int_{\Omega^{\prime}}|\vartheta|^{\frac{q q^{\prime}}{q^{\prime}-q}} \mathrm{~d} x\right)^{1-\frac{q}{q^{\prime}}}
\end{aligned}
$$


where we have applied Meyers' theorem to the term $\|\nabla F(\sigma+\vartheta)\|_{L_{q^{\prime}}\left(\Omega^{\prime}\right)}$. The choice $\frac{q q^{\prime}}{q^{\prime}-q} \geq p$ implies

$$
\int_{\Omega^{\prime}}|\vartheta|^{\frac{q q^{\prime}}{q^{\prime}-q}} \mathrm{~d} x=\int_{\Omega^{\prime}}|\vartheta|^{p} \cdot|\vartheta|^{\frac{q q^{\prime}}{q^{\prime}-q}-p} \mathrm{~d} x \leq|\lambda|^{p-\frac{q q^{\prime}}{q^{\prime}-q}} \int_{\Omega^{\prime}}|\vartheta|^{p} \mathrm{~d} x
$$

Collecting the exponents in inequalities (2.2)-(2.4) yields

$$
\|F(\sigma+\vartheta)-F(\sigma)\|_{W^{1, q}\left(\Omega^{\prime}\right)} \leq C\|\vartheta\|_{L_{p}\left(\Omega^{\prime}\right)}^{\frac{q^{\prime}-q}{q^{\prime} p}} .
$$

The choice of $p \in\left(\frac{4 Q(\lambda)}{Q(\lambda)-2}, \infty\right]$ indicates that $q \in\left(2, \frac{4 Q(\lambda)}{2+Q(\lambda)}\right) \subset(2, Q(\lambda))$, and thus $q<q^{\prime}=\frac{2 q}{4-q}<Q(\lambda)$. With this choice of $q^{\prime}$, we have $\frac{q^{\prime}-q}{q^{\prime} q} p=1$, i.e.,

$$
\|F(\sigma+\vartheta)-F(\sigma)\|_{W^{1, q}\left(\Omega^{\prime}\right)} \leq C\|\vartheta\|_{L_{p}\left(\Omega^{\prime}\right)},
$$

which shows the second assertion.

Now we turn to the third assertion. By the proof of the second assertion, for any $q \in(2, Q(\lambda))$ and $q^{\prime} \in$ $(q, Q(\lambda))$ we have

$$
\|\vartheta \nabla F(\sigma+\vartheta)\|_{L_{q}\left(\Omega^{\prime}\right)} \leq C\|j\|_{H^{-\frac{1}{2}}(\Gamma)}\|\vartheta\|_{L_{\frac{q q^{\prime}}{q^{\prime}-q}}\left(\Omega^{\prime}\right)} .
$$

If the exponent $\tilde{p}=\frac{q q^{\prime}}{q^{\prime}-q} \leq p$, then by Hölder's inequality, we have

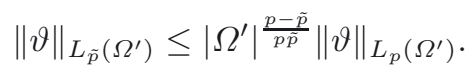

As to the other case, by the $L_{\infty}(\Omega)$ boundedness of the admissible set $\mathcal{A}$, we deduce for $\tilde{p} \geq p \geq 1$

$$
\|\vartheta\|_{L_{\tilde{p}}\left(\Omega^{\prime}\right)}^{\tilde{p}} \leq C\|\vartheta\|_{L_{p}\left(\Omega^{\prime}\right)}^{p} .
$$

In either case, we have that the term goes to zero as $\|\vartheta\|_{L_{p}\left(\Omega^{\prime}\right)} \rightarrow 0$. Hence, we obtain

$$
\lim _{\|\vartheta\|_{L_{p}\left(\Omega^{\prime}\right)} \rightarrow 0}\|F(\sigma+\vartheta)-F(\sigma)\|_{W^{1, q}\left(\Omega^{\prime}\right)}=0
$$

for arbitrary $p \geq 1$.

Remark 2.6. For the case of $p \in\left(\frac{2 Q(\lambda)}{Q(\lambda)-2}, \frac{4 Q(\lambda)}{Q(\lambda)-2}\right)$ and $\frac{1}{q}=\frac{1}{2}-\frac{1}{p}$, we have by choosing $q^{\prime}=Q(\lambda)-\epsilon$

$$
\|F(\sigma+\vartheta)-F(\sigma)\|_{W^{1, q}\left(\Omega^{\prime}\right)} \leq C\|\vartheta\|_{L_{p}\left(\Omega^{\prime}\right)}^{r} \quad \text { with } \quad r=\frac{2(Q(\lambda)-\epsilon-q)}{(Q(\lambda)-\epsilon)(q-2)}
$$

It is easy to see that $r<\frac{2 Q(\lambda)-q}{Q(\lambda)(q-2)} \leq 1$.

Let us now proceed to the differentiability of the forward operator $F(\sigma)$. We fix $\sigma \in \mathcal{A}$, and let $\vartheta$ be a perturbation to $\sigma$ belonging to $L_{\infty}\left(\Omega^{\prime}\right)$ and extended by zero outside $\Omega^{\prime}$. Let $w \in \tilde{H}^{1}(\Omega)$ be the weak solution to

$$
\int_{\Omega} \sigma \nabla w \cdot \nabla v \mathrm{~d} x=-\int_{\Omega} \vartheta \nabla F(\sigma) \cdot \nabla v \mathrm{~d} x \quad \forall v \in \tilde{H}^{1}(\Omega)
$$

The above equation is the linearized problem of the Neumann forward problem at $\sigma$. We shall call $F^{\prime}(\sigma)$ : $L_{p}\left(\Omega^{\prime}\right) \rightarrow \tilde{H}^{1}(\Omega)$ the map from $\vartheta$ to $w$.

Lemma 2.7. For any $\sigma \in \mathcal{A}$, the linear map $F^{\prime}(\sigma)$ defined above has the following continuity properties 
(a) For any $p \in\left(\frac{2 Q(\lambda)}{Q(\lambda)-2}, \infty\right]$, the operator $F^{\prime}(\sigma): L_{p}\left(\Omega^{\prime}\right) \rightarrow \tilde{H}^{1}(\Omega)$ is bounded;

(b) for any $p \in\left(\frac{4 Q(\lambda)}{Q(\lambda)-2}, \infty\right]$, there exists a $q \in(2, Q(\lambda))$ such that $F^{\prime}(\sigma): L_{p}\left(\Omega^{\prime}\right) \rightarrow W^{1, q}\left(\Omega^{\prime}\right)$ is bounded;

(c) for $p \geq 1$ and any $q \in(2, Q(\lambda))$

$$
\lim _{\|\vartheta\|_{L_{p}\left(\Omega^{\prime}\right)} \rightarrow 0}\left\|F^{\prime}(\sigma) \vartheta\right\|_{W^{1, q}\left(\Omega^{\prime}\right)}=0 .
$$

Proof. For any $p \in\left(\frac{2 Q(\lambda)}{Q(\lambda)-2}, \infty\right]$, we can choose $q$ by $\frac{1}{q}+\frac{1}{p}=\frac{1}{2}$, i.e., $q \in(2, Q(\lambda))$. By the weak formulation of $F^{\prime}(\sigma) \vartheta$ and the generalized Hölder inequality, we have

$$
\begin{aligned}
\int_{\Omega} \sigma\left|\nabla F^{\prime}(\sigma) \vartheta\right|^{2} \mathrm{~d} x & =-\int_{\Omega} \vartheta \nabla F(\sigma) \cdot \nabla F^{\prime}(\sigma) \vartheta \mathrm{d} x \\
& =-\int_{\Omega^{\prime}} \vartheta \nabla F(\sigma) \cdot \nabla F^{\prime}(\sigma) \theta \mathrm{d} x \\
& \leq\|\vartheta\|_{L_{p}\left(\Omega^{\prime}\right)}\|\nabla F(\sigma)\|_{L_{q}\left(\Omega^{\prime}\right)}\left\|\nabla F^{\prime}(\sigma) \vartheta\right\|_{L_{2}(\Omega)} \\
& \leq C\|\vartheta\|_{L_{p}\left(\Omega^{\prime}\right)}\|F(\sigma)\|_{H^{1}(\Omega)}\left\|\nabla F^{\prime}(\sigma) \vartheta\right\|_{L_{2}(\Omega)}
\end{aligned}
$$

This together with Lemma 2.3 implies that the operator $F^{\prime}(\sigma): L_{p}\left(\Omega^{\prime}\right) \rightarrow \tilde{H}^{1}(\Omega)$ is bounded, thereby showing the first assertion. To prove the second and third assertions, we appeal to Meyers' theorem to derive

$$
\left\|F^{\prime}(\sigma) \vartheta\right\|_{W^{1, q}\left(\Omega^{\prime}\right)} \leq C\left(\left\|F^{\prime}(\sigma) \vartheta\right\|_{H^{1}(\Omega)}+\|\vartheta \nabla F(\sigma)\|_{L_{q}(\Omega)}\right) .
$$

Therefore, we need to bound the term $\|\vartheta \nabla F(\sigma)\|_{L_{q}(\Omega)}$, which can be estimated as in Lemma 2.5. This shows assertions (b) and (c).

The next result shows the differentiability of the operator $F(\sigma)$.

Theorem 2.8. Let $p \in\left(\frac{2 Q(\lambda)}{Q(\lambda)-2}, \infty\right]$. Then the forward operator $F(\sigma)$ is differentiable in the sense that for any $\sigma, \sigma+\vartheta \in \mathcal{A}$ there holds

$$
\frac{\left\|F(\sigma+\vartheta)-F(\sigma)-F^{\prime}(\sigma) \vartheta\right\|_{H^{1}(\Omega)}}{\|\vartheta\|_{L_{p}\left(\Omega^{\prime}\right)}} \rightarrow 0 \text { as } \vartheta \rightarrow 0 \text { in } L_{p}\left(\Omega^{\prime}\right) .
$$

Proof. Since $\vartheta$ vanishes on the boundary, the function $w=F(\sigma+\vartheta)-F(\sigma)-F^{\prime}(\sigma) \vartheta \in \tilde{H}^{1}(\Omega)$ satisfies

$$
\int_{\Omega}(\sigma+\vartheta) \nabla w \cdot \nabla v \mathrm{~d} x=-\int_{\Omega} \vartheta \nabla F^{\prime}(\sigma) \vartheta \cdot \nabla v \mathrm{~d} x \quad \forall v \in \tilde{H}^{1}(\Omega) .
$$

Taking $v=w$ in the weak formulation gives

$$
\begin{aligned}
\int_{\Omega}(\sigma+\vartheta)|\nabla w|^{2} \mathrm{~d} x & =-\int_{\Omega} \vartheta \nabla F^{\prime}(\sigma) \vartheta \cdot \nabla w \mathrm{~d} x=-\int_{\Omega^{\prime}} \vartheta \nabla F^{\prime}(\sigma) \vartheta \cdot \nabla w \mathrm{~d} x \\
& \leq\|\vartheta\|_{L_{p}\left(\Omega^{\prime}\right)}\left\|\nabla F^{\prime}(\sigma) \vartheta\right\|_{L_{q}\left(\Omega^{\prime}\right)}\|\nabla w\|_{L_{2}\left(\Omega^{\prime}\right)} .
\end{aligned}
$$

We observe that $p \in\left(\frac{2 Q(\lambda)}{Q(\lambda)-2}, \infty\right]$ implies $2 \leq q<Q(\lambda)$. By applying Lemma $2.7(\mathrm{c})$ to the term $\left\|\nabla F^{\prime}(\sigma) \vartheta\right\|_{L_{q}\left(\Omega^{\prime}\right)}$, we arrive at the desired assertion.

The Lipschitz continuity of the operator $F^{\prime}(\sigma)$ is essential, e.g., in studying convergence rates of regularization methods [19] and in analyzing iterative algorithms, and it will be used in Section 4.

Theorem 2.9. For any $p \in\left(\frac{4 Q(\lambda)}{Q(\lambda)-2}, \infty\right]$, the operator $F^{\prime}(\sigma)$ is Lipschitz continuous with respect to $L_{p}\left(\Omega^{\prime}\right)$ in the sense that for any $\sigma, \sigma+\vartheta \in \mathcal{A}$

$$
\left\|F^{\prime}(\sigma+\vartheta)-F^{\prime}(\sigma)\right\|_{L\left(L_{p}\left(\Omega^{\prime}\right), \tilde{H}^{1}(\Omega)\right)} \leq C\|\vartheta\|_{L_{p}\left(\Omega^{\prime}\right)} .
$$


Proof. For any $\varsigma \in L_{p}\left(\Omega^{\prime}\right)$, by the weak formulations for $F^{\prime}(\sigma) \varsigma$ and $F^{\prime}(\sigma+\vartheta) \varsigma$, i.e.,

$$
\begin{gathered}
\int_{\Omega} \sigma \nabla F^{\prime}(\sigma) \varsigma \cdot \nabla v \mathrm{~d} x=-\int_{\Omega} \varsigma \nabla F(\sigma) \cdot \nabla v \mathrm{~d} x \quad \forall v \in \tilde{H}^{1}(\Omega), \\
\int_{\Omega}(\sigma+\vartheta) \nabla F^{\prime}(\sigma+\vartheta) \varsigma \cdot \nabla v \mathrm{~d} x=-\int_{\Omega} \varsigma \nabla F(\sigma+\vartheta) \cdot \nabla v \mathrm{~d} x \quad \forall v \in \tilde{H}^{1}(\Omega),
\end{gathered}
$$

we derive that $w=F^{\prime}(\sigma+\vartheta) \varsigma-F^{\prime}(\sigma) \varsigma \in \tilde{H}^{1}(\Omega)$ satisfies

$$
\int_{\Omega} \sigma \nabla w \cdot \nabla v \mathrm{~d} x=-\int_{\Omega} \varsigma \nabla(F(\sigma+\vartheta)-F(\sigma)) \cdot \nabla v \mathrm{~d} x-\int_{\Omega} \vartheta \nabla F^{\prime}(\sigma+\vartheta) \varsigma \cdot \nabla v \mathrm{~d} x \quad \forall v \in \tilde{H}^{1}(\Omega) .
$$

Letting $v=w$ and applying the generalized Hölder's inequality with $q$ satisfying $\frac{1}{p}+\frac{1}{q}=\frac{1}{2}$ and $q \in(2, Q(\lambda))$ to the two terms on the right hand side of the above identity, we get

$$
\begin{aligned}
-\int_{\Omega} \varsigma \nabla(F(\sigma+\vartheta)-F(\sigma)) \cdot \nabla w \mathrm{~d} x & \leq\|\varsigma\|_{L_{p}\left(\Omega^{\prime}\right)}\|\nabla(F(\sigma+\vartheta)-F(\sigma))\|_{L_{q}\left(\Omega^{\prime}\right)}\|\nabla w\|_{L_{2}\left(\Omega^{\prime}\right)} \\
& \leq C\|\varsigma\|_{L_{p}\left(\Omega^{\prime}\right)}\|\vartheta\|_{L_{p}\left(\Omega^{\prime}\right)}\|\nabla w\|_{L_{2}\left(\Omega^{\prime}\right)},
\end{aligned}
$$

and

$$
\begin{aligned}
-\int_{\Omega} \vartheta \nabla F^{\prime}(\sigma+\vartheta) \varsigma \cdot \nabla w \mathrm{~d} x & \leq\|\vartheta\|_{L_{p}\left(\Omega^{\prime}\right)}\left\|\nabla F^{\prime}(\sigma+\vartheta) \varsigma\right\|_{L_{q}\left(\Omega^{\prime}\right)}\|\nabla w\|_{L_{2}\left(\Omega^{\prime}\right)} \\
& \leq C\|\varsigma\|_{L_{p}(\Omega)}\|\vartheta\|_{L_{p}\left(\Omega^{\prime}\right)}\|\nabla w\|_{L_{2}\left(\Omega^{\prime}\right)},
\end{aligned}
$$

in view of Lemmas 2.5(b) and 2.7(b), respectively. Combining these two estimates gives

$$
\|\nabla w\|_{L_{2}(\Omega)} \leq C\|\varsigma\|_{L_{p}\left(\Omega^{\prime}\right)}\|\vartheta\|_{L_{p}\left(\Omega^{\prime}\right)},
$$

which shows the desired assertion.

Remark 2.10. For any $p \in\left(\frac{4 Q(\lambda)}{Q(\lambda)-2}, \infty\right]$, by Theorem 2.9 and trace theorem [21], we have the following estimate for the linear approximation of the operator $F(\sigma)$

$$
\left\|F(\sigma+\vartheta)-F(\sigma)-F^{\prime}(\sigma) \vartheta\right\|_{L_{2}(\Gamma)} \leq \frac{L}{2}\|\vartheta\|_{L_{p}\left(\Omega^{\prime}\right)}^{2},
$$

where $L$ is the Lipschitz constant of the operator $F^{\prime}(\sigma)$, which depends on the constant from Meyers' estimate and the Sobolev embedding constant.

The adjoint of the operator $F^{\prime}(\sigma)$ is very useful in analyzing the convergence rate as well as in deriving the gradient of the discrepancy functional. We have the following representation, where $p^{*}$ is the conjugate exponent of $p \geq 1$, i.e., $\frac{1}{p}+\frac{1}{p^{*}}=1$.

Theorem 2.11. The adjoint of the operator $F^{\prime}(\sigma): L_{p}\left(\Omega^{\prime}\right) \rightarrow L_{2}(\Gamma)$ is given by

$$
\begin{aligned}
\left(F^{\prime}(\sigma)\right)^{*}: L_{2}(\Gamma) & \rightarrow L_{p^{*}}\left(\Omega^{\prime}\right), \\
f & \mapsto-\nabla \tilde{u} \cdot \nabla F(\sigma),
\end{aligned}
$$

where $\tilde{u} \in \tilde{H}^{1}(\Omega)$ solves the adjoint problem

$$
\int_{\Omega} \sigma \nabla \tilde{u} \cdot \nabla v \mathrm{~d} x=\int_{\Gamma} f v \mathrm{~d} s \quad \forall v \in \tilde{H}^{1}(\Omega) .
$$


Proof. For any $\vartheta \in L_{p}\left(\Omega^{\prime}\right)$, letting $v=\tilde{u}$ and $v=F^{\prime}(\sigma) \vartheta$ in the weak formulations for $F^{\prime}(\sigma) \vartheta$ and $\tilde{u}$ respectively gives

$$
\int_{\Omega^{\prime}}-\vartheta \nabla F(\sigma) \cdot \nabla \tilde{u} \mathrm{~d} x=\int_{\Gamma} F^{\prime}(\sigma) \vartheta f \mathrm{~d} s
$$

which shows the desired assertion.

In summary, we have arrived at the following useful corollary under the assumption:

Assumption 2.12. The dimension $d$ is $d=2$, or $d=3$ and additionally $\lambda$ is sufficiently close to one.

The closeness of $\lambda$ to one for $d=3$ is due to the finite embedding exponent of $H^{1}\left(\Omega^{\prime}\right)$ into $L_{p}\left(\Omega^{\prime}\right)$.

Corollary 2.13. Under Assumption 2.12, the operator $F(\sigma)$ is differentiable, and the operator $F^{\prime}(\sigma)$ is Lipschitz continuous with respect to the topology of $H^{1}\left(\Omega^{\prime}\right)$.

Proof. By the Sobolev embedding theorem [21], we have

$$
H^{1}\left(\Omega^{\prime}\right) \hookrightarrow L_{s}\left(\Omega^{\prime}\right) \text { for any }\left\{\begin{array}{l}
s<\infty, d=2, \\
s \leq 6, d=3 .
\end{array}\right.
$$

Therefore the result holds naturally for $d=2$. In case of $d=3$, we need some $Q(\lambda)>2$ such that $\frac{4 Q(\lambda)}{Q(\lambda)-2}<6$, i.e. $Q(\lambda)>6$, according to Lemma 2.5 and Theorem 2.9. By Meyers' theorem, we have $Q(\lambda) \rightarrow \infty$ as $\lambda \rightarrow 1$ and $Q(\lambda)$ depends continuously on $\lambda$ [48]. Therefore for $\lambda$ sufficiently close to 1 , we have $Q(\lambda)>6$ as desired.

Remark 2.14. Note that the classical $L_{\infty}$ estimates do not imply Corollary 2.13 since $H^{1}\left(\Omega^{\prime}\right)$ does not embed continuously into $L_{\infty}\left(\Omega^{\prime}\right)$ for $d=2,3$, and thus the $L_{p}$ estimates derived here are advantageous for justifying regularization in the Hilbert space $H^{1}\left(\Omega^{\prime}\right)$.

The continuity and differentiability results can be used to study various discrepancy functionals. The following result shows the continuity of the standard least-squares discrepancy $J(\sigma)=\frac{1}{2}\left\|F(\sigma)-\phi^{\delta}\right\|_{L_{2}(\Gamma)}^{2}$.

Proposition 2.15. The functional $J$ is Hölder continuous with respect to $L_{p}\left(\Omega^{\prime}\right)$ for any $1 \leq p \leq \infty$.

Proof. First we fixed $p \in\left(\frac{2 Q(\lambda)}{Q(\lambda)-2}, \infty\right]$. For any $\sigma, \sigma+\vartheta \in \mathcal{A}$, by Cauchy-Schwarz inequality, we observe

$$
|J(\sigma+\vartheta)-J(\sigma)| \leq \frac{1}{2}\|F(\sigma)-F(\sigma+\vartheta)\|_{L_{2}(\Gamma)}\left\|F(\sigma)+F(\sigma+\vartheta)-2 \phi^{\delta}\right\|_{L_{2}(\Gamma)} .
$$

The choice $p \in\left(\frac{2 Q(\lambda)}{Q(\lambda)-2}, \infty\right]$ implies the existence of a $q \in(2, Q(\lambda))$ such that $\frac{1}{p}+\frac{1}{q}=\frac{1}{2}$. By trace theorem [21], Lemma 2.5 and the generalized Hölder inequality, we can estimate the term $\|F(\sigma+\vartheta)-F(\sigma)\|_{L_{2}(\Gamma)}$ as

$$
\|F(\sigma)-F(\sigma+\vartheta)\|_{L_{2}(\Gamma)} \leq C\|F(\sigma+\vartheta)-F(\sigma)\|_{H^{1}(\Omega)} \leq C\|\vartheta\|_{L_{p}\left(\Omega^{\prime}\right)} .
$$

The term $\left\|F(\sigma)+F(\sigma+\vartheta)-2 \phi^{\delta}\right\|_{L_{2}(\Gamma)}$ is uniformly bounded by Lemma 2.2 and trace theorem. Therefore, $J$ is Lipschitz continuous with respect to $L_{p}\left(\Omega^{\prime}\right)$ for any $p \in\left(\frac{2 Q(\lambda)}{Q(\lambda)-2}, \infty\right]$. The Hölder continuity with respect to $L_{p}\left(\Omega^{\prime}\right)$ for any $1 \leq p \leq \infty$ follows from the uniform $L_{\infty}(\Omega)$ boundedness of the set $\mathcal{A}$.

Remark 2.16. All the results presented so far are for the admissible set $\mathcal{A}$, which allows only variation of the conductivity in the interior part $\Omega^{\prime}$ of the domain $\Omega$. This restriction can be removed by imposing higher regularity on the flux $j$, i.e., $j \in L_{s}(\Gamma) \cap \tilde{H}^{-\frac{1}{2}}(\Gamma)$ for sufficiently large $s$, and on the boundary $\Gamma$, see Theorem 3.1 in Section 3. All the results remain valid with this modification. 


\section{Complete electrode model}

This section discusses relevant analytical results for the complete electrode model (CEM), presently the most accurate model. This model can achieve an accuracy comparable with experimental precision [11, 12,53], and thus it is standard model for medical applications.

In contrast to the continuum model discussed earlier, the CEM utilizes nonstandard boundary conditions to capture essential features of EIT experiments, e.g., discrete nature of electrodes, contact impedance effect and shunting effect. Let $\Omega \subset \mathbb{R}^{d}(d=2,3)$ be an open bounded domain with a smooth boundary, and $\left\{e_{l}\right\}_{l=1}^{L} \subset \Gamma$ be $L$ electrodes, each with a positive surface measure. We assume that each electrode is connected and they are disjointed from each other, i.e., $\bar{e}_{i} \cap \bar{e}_{j}=\emptyset$ for $i \neq j$. Let $\mathbb{R}_{\diamond}^{L}:=\left\{I \in \mathbb{R}^{L}: \sum_{l=1}^{L} I_{l}=0\right\}$, and $H=H^{1}(\Omega) \oplus \mathbb{R}_{\diamond}^{L}$ with its norm defined by $\|(v, V)\|_{H}^{2}=\|v\|_{H^{1}(\Omega)}^{2}+\|V\|_{\mathbb{R}^{L}}^{2}$, which is equivalent to the norm defined by $\|(v, V)\|_{*}^{2}=$ $\|\nabla v\|_{L_{2}(\Omega)}^{2}+\sum_{l=1}^{L}\left\|v-V_{l}\right\|_{L_{2}\left(e_{l}\right)}^{2}[53]$. Then the model reads as follows: given an input current $I \in \mathbb{R}_{\diamond}^{L}$ and a set of positive contact impedances $\left\{z_{l}\right\}_{l=1}^{L}$, find $(u, U) \in H$ such that

$$
\begin{cases}-\nabla \cdot(\sigma \nabla u)=0 & \text { in } \Omega, \\ u+z_{l} \sigma \frac{\partial u}{\partial n}=U_{l} & \text { on } e_{l}, l=1,2, \ldots, L \\ \int_{e_{l}} \sigma \frac{\partial u}{\partial n} \mathrm{~d} s=I_{l} & \text { for } l=1,2, \ldots, L \\ \sigma \frac{\partial u}{\partial n}=0 & \text { on } \Gamma \backslash \cup_{l=1}^{L} e_{L} .\end{cases}
$$

Then the inverse problem is to estimate the conductivity parameter $\sigma$ from the measured voltage vector $U \in$ $\mathbb{R}_{\diamond}^{L}$. We note that, theoretically, the (finite-dimensional) current-to-voltage map can be regarded as a discrete approximation of the Dirichlet-to-Neumann map of the continuum model as the electrodes get infinitesimally small and cover the whole boundary $\Gamma[29]$.

We first discuss the forward problem (3.1). The weak formulation is given by [53]: find $(u, U) \in H$ such that

$$
\int_{\Omega} \sigma \nabla u \cdot \nabla v \mathrm{~d} x+\sum_{l=1}^{L} z_{l}^{-1} \int_{e_{l}}\left(u-U_{l}\right)\left(v-V_{l}\right) \mathrm{d} s=\sum_{l=1}^{L} I_{l} V_{l} \quad \forall(v, V) \in H .
$$

Lax-Milgram theorem yields directly the existence and uniqueness of a solution $(u, U)[53]$. We denote the solution operator by $F(\sigma)$, i.e., $(u, U)=\left(F_{1}(\sigma) I, F_{2}(\sigma) I\right)=F(\sigma) I \in H$. Again, we suppress the dependence of the solution $(u, U)$ on the input current $I$. The admissible set $\mathcal{A}$ for the conductivity $\sigma$ is given by $\mathcal{A}=\{\sigma \in$ $L_{\infty}(\Omega): \lambda \leq \sigma \leq \lambda^{-1}$ a.e. $\left.\Omega\right\}$ for some fixed constant $\lambda \in(0,1)$.

We first recall the following elliptic regularity estimate [22], Theorem 2, [25], which is analogue of Theorem 2.4 for Neumann problems. It enables exploiting the higher regularity of the Neumann boundary condition in system (3.1) and thus establishing better regularity for the CEM model.

Theorem 3.1. For any $\sigma \in \mathcal{A}$, there exists a constant $Q$, which depends on $d$ and $\lambda$ only and tends to $\infty$ and 2 as $\lambda \rightarrow 1$ and $\lambda \rightarrow 0$, respectively, such that for any $q \in(2, Q)$, any $s \in\left[q-\frac{q}{d}, \infty\right]$ and $j \in L_{s}(\Gamma) \cap \tilde{H}^{-\frac{1}{2}}(\Gamma)$, $f \in\left(L_{q}(\Omega)\right)^{d}$, the solution $u$ to the Neumann problem

$$
-\nabla \cdot(\sigma \nabla u)=\nabla \cdot f \quad \text { in } \Omega \quad \text { and } \quad \sigma \frac{\partial u}{\partial n}=j \quad \text { on } \Gamma
$$

satisfies the estimate

$$
\|u\|_{W^{1, q}(\Omega)} \leq C\left(\|j\|_{L_{s}(\Gamma)}+\|f\|_{L_{q}(\Omega)}\right),
$$

where $C$ is a constant depending on $d, \lambda, \Omega$ and $q$ only.

A first estimate is the uniform boundedness of the operator $F(\sigma)$. 
Lemma 3.2. The operator $F(\sigma): \mathcal{A} \rightarrow H$ is uniformly bounded.

Proof. Setting $(v, V)=(u, U) \in H$ in the weak formulation (3.2) gives

$$
\begin{aligned}
\lambda\|\nabla u\|_{L_{2}(\Omega)}^{2}+c_{0} \sum_{l=1}^{L}\left\|u-U_{l}\right\|_{L_{2}\left(e_{l}\right)}^{2} & \leq \int_{\Omega} \sigma|\nabla u|^{2} \mathrm{~d} x+\sum_{l=1}^{L} z_{l}^{-1} \int_{e_{l}}\left(u-U_{l}\right)^{2} \mathrm{~d} s \\
& =\sum_{l=1}^{L} I_{l} U_{l} \leq\|I\|_{\mathbb{R}^{L}}\|(u, U)\|_{H},
\end{aligned}
$$

where $c_{0}=\min \left\{z_{l}^{-1}, l=1, \ldots, L\right\}$. This shows the uniform boundedness of the operator $F(\sigma)$.

The following theorem provides the key regularity result.

Theorem 3.3. For any $\sigma \in \mathcal{A}$, there exists a constant $Q>2$, depending on $d$ and $\lambda$ only and tending to $\infty$ and 2 as $\lambda \rightarrow 1$ and $\lambda \rightarrow 0$, respectively, such that the solution $(u, U) \in H$ to (3.1) satisfies for any $q \in(2, Q(\lambda))$

$$
\|u\|_{W^{1, q}(\Omega)} \leq C\|I\|_{\mathbb{R}^{L}},
$$

where $C$ is a constant depending on $d, \lambda, \Omega$ and $q$ only.

Proof. By Lemma 3.2, there exists a solution $(u, U) \in H$ such that

$$
\|(u, U)\|_{H} \leq C\|I\|_{\mathbb{R}^{L}} .
$$

Next we rewrite equation (3.1) as

$$
\begin{cases}-\nabla \cdot(\sigma \nabla u)=0 & \text { in } \Omega \\ \sigma \frac{\partial u}{\partial n}=g, & \text { on } \Gamma,\end{cases}
$$

where the function $g=\sum_{l=1}^{L} \frac{1}{z_{l}}\left(U_{l}-u\right) \chi_{e_{l}} \in \tilde{H}^{-\frac{1}{2}}(\Gamma)$ (the fact that $g \in \tilde{H}^{-\frac{1}{2}}(\Gamma)$ follows from (3.2)). Note that $u \in H^{1}(\Omega)$ and $U_{l}$ is a constant. By Sobolev embedding theorem [21], we have $g \in L_{s}(\Gamma), \forall s<\infty$ if $d=2$ and $g \in L_{4}(\Gamma)$ if $d=3$. In the case of $d=2$, by Theorem 3.1, we have for any $q<Q(\lambda)$

$$
\|\nabla u\|_{L_{q}(\Omega)} \leq C\|g\|_{L_{s}(\Gamma)} \leq C\|I\|_{\mathbb{R}^{L}},
$$

by Lemma 3.2. In the case of $d=3$, similarly by Theorem 3.1, we have for any $q<\min (Q(\lambda), 6)$ again $\|\nabla u\|_{L_{q}(\Omega)} \leq C\|I\|_{\mathbb{R}^{L}}$ holds. The proof of the theorem is concluded if $Q(\lambda)<6$, otherwise we can repeat the procedure with the estimate $\|\nabla u\|_{L_{q}(\Omega)} \leq C\|I\|_{\mathbb{R}^{L}}$ for $q<6$ and Sobolev embedding theorem [21] that $W^{1, r}(\Omega)$ with $r>3$ embeds continuously into $L_{\infty}(\Gamma)$, and the theorem follows.

With Theorem 3.3 at hand, we can state analogous continuity and differentiability results for the CEM forward operator $F(\sigma)$. The proofs are identical with those in Section 2, and thus omitted for clarity. A first result is the following continuity result.

Lemma 3.4. For the operator $F(\sigma)$ and $\sigma, \sigma+\vartheta \in \mathcal{A}$, we have the following continuity properties.

(a) For any $p \in\left(\frac{2 Q(\lambda)}{Q(\lambda)-2}, \infty\right]$ and $\sigma, \sigma+\vartheta \in \mathcal{A}$ we have

$$
\|F(\sigma+\vartheta)-F(\sigma)\|_{H} \leq C\|\vartheta\|_{L_{p}(\Omega)}
$$

(b) for any $p \in\left(\frac{4 Q(\lambda)}{Q(\lambda)-2}, \infty\right]$, there exists a $q \in(2, Q(\lambda))$ such that

$$
\left\|F_{1}(\sigma+\vartheta)-F_{1}(\sigma)\right\|_{W^{1, q}(\Omega)} \leq C\|\vartheta\|_{L_{p}(\Omega)} ;
$$


(c) for $p \geq 1$ and any $q \in(2, Q(\lambda))$ we have the following estimates

$$
\lim _{\|\vartheta\|_{L_{p}(\Omega)} \rightarrow 0}\left\|F_{1}(\sigma+\vartheta)-F_{1}(\sigma)\right\|_{W^{1, q}(\Omega)}=0 .
$$

Let us now proceed to differentiability. As before, we fix $\sigma \in \mathcal{A}$, and let $\vartheta$ be a perturbation to $\sigma$ belonging to $L_{\infty}(\Omega)$. Let $(w, W) \in H$ be the weak solution to

$$
\int_{\Omega} \sigma \nabla w \cdot \nabla v \mathrm{~d} x+\sum_{l=1}^{L} z_{l}^{-1} \int_{e_{l}}\left(w-W_{l}\right)\left(v-V_{l}\right) \mathrm{d} s=-\int_{\Omega} \vartheta \nabla F_{1}(\sigma) \cdot \nabla v \mathrm{~d} x \quad \forall(v, V) \in H .
$$

The above equation is the linearized problem of the CEM forward problem at $\sigma$. We shall call $F^{\prime}(\sigma): L_{p}(\Omega) \rightarrow H$ the map from $\vartheta$ to $(w, W)$. Then we have the following.

Lemma 3.5. For any $\sigma \in \mathcal{A}$, the linear map $F^{\prime}(\sigma)$ defined above has the following continuity properties.

(a) For any $p \in\left(\frac{2 Q(\lambda)}{Q(\lambda)-2}, \infty\right]$, the operator $F^{\prime}(\sigma): L_{p}(\Omega) \rightarrow H$ is bounded;

(b) for any $p \in\left(\frac{4 Q(\lambda)}{Q(\lambda)-2}, \infty\right]$, there exists $q \in(2, Q(\lambda))$ such that $F_{1}^{\prime}(\sigma): L_{p}(\Omega) \rightarrow W^{1, q}(\Omega)$ is bounded;

(c) for $p \geq 1$ and any $q \in(2, Q(\lambda))$

$$
\lim _{\|\vartheta\|_{L_{p}(\Omega)} \rightarrow 0}\left\|F_{1}^{\prime}(\sigma) \vartheta\right\|_{W^{1, q}(\Omega)}=0 .
$$

Now we can state differentiability of the operator $F(\sigma)$, and Lipschitz continuity of the operator $F^{\prime}(\sigma)$.

Theorem 3.6. For any $\sigma, \sigma+\vartheta \in \mathcal{A}$, there hold

(a) For any $p \in\left(\frac{2 Q(\lambda)}{Q(\lambda)-2}, \infty\right]$, the operator $F(\sigma)$ is differentiable in the sense

$$
\frac{\left\|F(\sigma+\vartheta)-F(\sigma)-F^{\prime}(\sigma) \vartheta\right\|_{H}}{\|\vartheta\|_{L_{p}(\Omega)}} \rightarrow 0 \text { as } \vartheta \rightarrow 0 \text { in } L_{p}(\Omega) ;
$$

(b) for any $p \in\left(\frac{4 Q(\lambda)}{Q(\lambda)-2}, \infty\right]$, the operator $F^{\prime}(\sigma)$ is Lipschitz continuous in the sense

$$
\left\|F^{\prime}(\sigma+\vartheta)-F^{\prime}(\sigma)\right\|_{L\left(L_{p}(\Omega), H\right)} \leq C\|\vartheta\|_{L_{p}(\Omega)} .
$$

As to the adjoint of the operator $F^{\prime}(\sigma): L_{p}(\Omega) \rightarrow \mathbb{R}_{\diamond}^{L}$, we have the following representation.

Theorem 3.7. The adjoint of the operator $F^{\prime}(\sigma): L_{p}(\Omega) \rightarrow \mathbb{R}_{\diamond}^{L}$ is given by

$$
\begin{aligned}
\left(F^{\prime}(\sigma)\right)^{*}: \mathbb{R}_{\diamond}^{L} & \rightarrow L_{p^{*}}(\Omega), \\
\tilde{I} & \mapsto-\nabla \tilde{u} \cdot \nabla F_{1}(\sigma),
\end{aligned}
$$

where $(\tilde{u}, \tilde{U}) \in H$ solves the adjoint problem

$$
\int_{\Omega} \sigma \nabla \tilde{u} \cdot \nabla v \mathrm{~d} x+\sum_{l=1}^{L} z_{l}^{-1} \int_{e_{l}}\left(\tilde{u}-\tilde{U}_{l}\right)\left(v-V_{l}\right) \mathrm{d} s=\sum_{l=1}^{L} \tilde{I}_{l} V_{l} \quad \forall(v, V) \in H .
$$

In summary, we have the following corollary for the CEM forward operator $F(\sigma)$.

Corollary 3.8. If $d=2$, or if $d=3$ and additionally $\lambda$ is sufficiently close to one, then the operator $F(\sigma)$ is differentiable, and the operator $F^{\prime}(\sigma)$ is Lipschitz continuous with respect to the topology of $H^{1}(\Omega)$. 


\section{Applications to smoothness/Sparsity Regularization}

Now we apply the analytical results of the previous sections to investigate several Tikhonov functionals for the EIT inverse problem. The penalties of interest include smoothness and sparsity penalties. The former has been very popular, while the latter has demonstrated its potential only recently $[23,36]$.

Due to its relatively recent origin, we would like to briefly describe the sparsity approach. Given an orthonormal basis/overcomplete frame $\left\{\psi_{k}\right\}$, a signal is called sparse if the sequence of expansion coefficients with respect to $\left\{\psi_{k}\right\}$ contains only a finite (or small) number of nonzero entries. Such a prior knowledge can be effectively used for encoding and decoding signals with far less data via $\ell_{1}$ programming [17]. In the context of EIT, sparsity assumption is plausible for conductivity fields that consist of an unknown but essentially uninteresting background plus a number of interesting features that have relatively "simple" mathematical descriptions. For instance, inclusions have a sparse representation in pixel basis, and piecewise constant conductivities have sparse representations in wavelet basis. Recently, a novel reconstruction algorithm of iterative soft shrinkage type has been developed [36], based on approximately minimizing a Tikhonov functional with the sparsity penalty, see [36] for intricacies of its efficient implementation. The simulation and experimental results in [36] and [23] show that sparsity constraint represents one powerful imaging technique in that it is capable of reconstructing nonconvex/multiple inclusions with correct magnitudes.

The purpose of incorporating a priori knowledge such as smoothness or sparsity is to counter insufficient amount of information in the data as well as to combat ill-posed nature of the problem. We are interested in imaging conductivity fields that away from a known background $\sigma_{0}$ are either smooth or sparse. Let $\vartheta=\sigma-\sigma_{0}$ be the inclusion/inhomogeneity. Here the background $\sigma_{0}$ can be quite arbitrary, e.g., discontinuous. The setting we are going to use for $\vartheta$ is a Hilbert space $H_{0}^{1}\left(\Omega^{\prime}\right)$, i.e., $\vartheta \in H_{0}^{1}\left(\Omega^{\prime}\right)$, and we equip the space $H_{0}^{1}\left(\Omega^{\prime}\right)$ with an orthonormal basis $\left\{\psi_{k}\right\}$. Then on the sequence $\left\{\left\langle\vartheta, \psi_{k}\right\rangle\right\}$ of expansion coefficients, we endow $\ell_{r}$ norms, i.e.,

$$
\|\vartheta\|_{\ell_{r}}^{r}=\sum_{k=1}^{\infty}\left|\left\langle\vartheta, \psi_{k}\right\rangle\right|^{r} .
$$

We consider the following penalty $R_{r}(\vartheta)$

$$
R_{r}(\vartheta)=\frac{1}{r}\|\vartheta\|_{\ell_{r}}^{r} \quad 1 \leq r \leq 2 .
$$

Several remarks on the $R_{r}$ penalty are in order. First, we observe that the penalty $R_{r}(\vartheta)$ is convex and weakly lower semi-continuous [6]. Second, the choice $r=2$ reproduces the classical smoothness penalty, i.e., $R_{2}(\vartheta)=\frac{1}{2}\|\vartheta\|_{H^{1}\left(\Omega^{\prime}\right)}^{2}$ in view of the norm equivalence, which is one of most widely used penalties since the inaugural work [54], see [45] for a survey of its applications in EIT. Third, the choice $r \in[1,2)$ is motivated by sparsity constraint [14]. Here $\vartheta$ is assumed to have a sparse representation in the basis $\left\{\psi_{k}\right\}$, i.e., only finitely many coefficients $\left\{\left\langle\vartheta, \psi_{k}\right\rangle\right\}$ are nonzero. It is widely accepted that sparsity may be promoted via an $R_{r}(r \in[1,2))$, prominently $R_{1}$, penalty on expansion coefficients. Therefore, by considering an $R_{r}(r \in[1,2])$ penalty, we treat the smoothness/sparsity penalty in a unified way.

We study the linearized and fully nonlinear models separately, by capitalizing on recent progress on nonsmooth regularization $[5,8,24,28,34,35,46,50]$. The analysis of Tikhonov models relies mainly on the boundedness of the derivative operator $F^{\prime}(\sigma)$ and the differentiability of the forward operator $F(\sigma)$ in case of linearized and nonlinear models, respectively. Since these properties hold for both the continuum model in Section 2 and the complete electrode model in Section 3, we refrain from repeating the statements for both models and present only results for the continuum model. The complete electrode model can be treated analogously.

\subsection{Linearized model}

Although the EIT inverse problem is inherently nonlinear, a linearized model has been popular, see $[10,45,56,57]$ for a rather incomplete list. This is partly attributed to the fact that there are diverse sources, 
possibly significant, of model uncertainties, e.g., in geometry and boundary conditions. However, the analysis of such linearization procedure lags far behind, and many basic questions about the validity of the procedure remains unaddressed. Recently [26] shows that such a procedure preserves the outer support of the inclusions.

The linearized model consists of approximately solving the following operator equation

$$
F^{\prime}\left(\sigma_{0}\right) \vartheta+F\left(\sigma_{0}\right)-\phi^{\delta}=0 .
$$

For the analysis of (4.1), the operator $F^{\prime}\left(\sigma_{0}\right)$ should be bounded. Hence throughout this part, we require that Assumption 2.12 hold ( $c f$. Cor. 2.13). A first remark concerning the linearized problem (4.1) is as follows.

Remark 4.1. By Lemma 2.7 and Corollary 2.13, the linear operator $F^{\prime}(\sigma): H_{0}^{1}\left(\Omega^{\prime}\right) \rightarrow \tilde{H}^{1}(\Omega)$ is bounded, and by trace theorem [21], i.e., $\tilde{H}^{1}(\Omega)$ embeds compactly into $L_{2}(\Gamma)$, the map $F^{\prime}(\sigma): H_{0}^{1}\left(\Omega^{\prime}\right) \rightarrow L_{2}(\Gamma)$ is thus bounded and compact. Consequently, the linearized equation (4.1) is ill-posed. The analysis developed here does not cover the popular $T V$ penalty since the space of bounded variation $B V\left(\Omega^{\prime}\right)$ only embeds into $L_{\frac{d}{d-1}}\left(\Omega^{\prime}\right)[21]$, while the boundedness of $F^{\prime}(\sigma): L_{\frac{d}{d-1}}\left(\Omega^{\prime}\right) \rightarrow L_{2}(\Gamma)(d=2,3)$ is yet to be established.

Remark 4.2. The space $\ell_{r}(r \in[1,2))$ is a subspace of $\ell_{2}$, and thus the $R_{r}$-penalty enforces a stronger penalization than $H^{1}\left(\Omega^{\prime}\right)$-penalty.

According to Remark 4.1 the linear operator equation (4.1) is ill-posed. For its stable numerical solution, we consider Tikhonov regularization

$$
\Psi_{\mathrm{L}}(\vartheta)=\frac{1}{2}\left\|F^{\prime}\left(\sigma_{0}\right) \vartheta-\tilde{\phi}^{\delta}\right\|_{L_{2}(\Gamma)}^{2}+\alpha R_{r}(\vartheta)
$$

where $\tilde{\phi}^{\delta}=\phi^{\delta}-F\left(\sigma_{0}\right)$ denotes the linearized noisy data, and $\alpha$ is a scalar compromising the two terms.

A first question regarding any mathematical model is its well-posedness. In light of the analytical machineries in Section 2 and Remark 4.1, we have the following existence and stability results. Hence the model $\Phi_{\mathrm{L}}$ is indeed well-posed, which ensures the good numerical behavior of subsequent minimization procedures. The results address a linear inverse problem, and follow directly from the general theory in [14].

Theorem 4.3. There exists at least one minimizer $\vartheta_{\alpha}^{\delta}$ to the functional $\Psi_{\mathrm{L}}$. Let $\left\{\tilde{\phi}^{n}\right\} \subset L_{2}(\Gamma)$ be a sequence of noisy data converging to $\tilde{\phi}^{\delta}$, and $\vartheta^{n}$ be a minimizer to $\Psi_{\mathrm{L}}$ with $\tilde{\phi}^{n}$ in place of $\tilde{\phi}^{\delta}$. Then the sequence $\left\{\vartheta^{n}\right\}$ has a subsequence converging in $H^{1}\left(\Omega^{\prime}\right)$ to a minimizer of $\Psi_{\mathrm{L}}$.

To state a consistency result, we first recall the concept of an $R_{r}$-minimizing solution $\vartheta^{\dagger}$, i.e.,

$$
\vartheta^{\dagger}=\arg \min _{\vartheta \in \mathcal{S}} R_{r}(\vartheta)
$$

where the set $\mathcal{S}=\left\{\vartheta \in H_{0}^{1}\left(\Omega^{\prime}\right):\left\|F^{\prime}\left(\sigma_{0}\right) \vartheta-\tilde{\phi}^{\dagger}\right\|_{L_{2}(\Gamma)}=0\right\}$. We note that, generally, the set $\mathcal{S}$ contains multiple elements, and the $R_{r}$-minimizing solution $\vartheta^{\dagger}$ is not necessarily unique. If the linearized map $F^{\prime}\left(\sigma_{0}\right)$ is injective, then the set $\mathcal{S}$ consists of only one singleton and $\vartheta^{\dagger}$ is necessarily unique. This concept applies also to nonlinear models upon modifying $\mathcal{S}$ to $\mathcal{S}=\left\{\vartheta \in H_{0}^{1}\left(\Omega^{\prime}\right):\left\|F\left(\sigma_{0}+\vartheta\right)-\phi^{\dagger}\right\|_{L_{2}(\Gamma)}=0\right\}$, and the foregoing comments remain valid. In particular, if the given data is the complete Neumann-to-Dirichlet map, then it is expected that $\vartheta^{\dagger}$ is unique, in view of uniqueness solvability of the inverse problem, see [2] for the two-dimensional case.

The functional $\Psi_{\mathrm{L}}$ employs a linearized model, which represents the nonlinear model only approximately. It is unclear whether an exact solution for the noiseless data $\tilde{\phi}^{\dagger}=\phi^{\dagger}-F\left(\sigma_{0}\right)$ exists or not, i.e., it may lie beyond the range of $F^{\prime}\left(\sigma_{0}\right)$, which would result in an empty set $\mathcal{S}$. Then the set $\mathcal{S}$ may be redefined as parameters $\vartheta$ that attain a minimum of the residual. In order to avoid this complication, we a priori assume the existence of a solution of the linearized problem (for the data $\tilde{\phi}^{\dagger}$ ) as well as an $R_{r}$-minimizing solution. We note that $\vartheta^{\dagger}$ is generally different from the true inclusion $\sigma^{\dagger}-\sigma_{0}$.

Now we can state the following consistency result [14]. The consistency indicates that the minimizer $\vartheta_{\alpha}^{\delta}$ does approximate the true solution $\vartheta^{\dagger}$ if the noise in the data $\tilde{\phi}^{\delta}$ diminishes to zero. 
Theorem 4.4. If the parameter $\alpha=\alpha(\delta)$ satisfies $\lim _{\delta \rightarrow 0} \alpha(\delta)=0$ and $\lim _{\delta \rightarrow 0} \frac{\delta^{2}}{\alpha(\delta)}=0$, then the sequence of minimizers $\left\{\vartheta_{\alpha}^{\delta}\right\}$ has a convergent subsequence in $H^{1}\left(\Omega^{\prime}\right)$ to an $R_{r}$-minimizing solution $\vartheta^{\dagger}$ as $\delta \rightarrow 0$. Further, if $\vartheta^{\dagger}$ is unique, then the whole sequence converges.

In order to obtain quantitative estimates for the minimizer $\vartheta_{\alpha}^{\delta}$, additional conditions on the solution $\vartheta^{\dagger}$, are required. To this end, we need the adjoint of the operator $F^{\prime}(\sigma)$ with respect to the $H_{0}^{1}\left(\Omega^{\prime}\right)$ inner product.

Theorem 4.5. The adjoint of the operator $F^{\prime}(\sigma): H_{0}^{1}\left(\Omega^{\prime}\right) \rightarrow L_{2}(\Gamma)$ is given by

$$
\begin{aligned}
\left(F^{\prime}(\sigma)\right)^{*}: L_{2}(\Gamma) & \rightarrow H_{0}^{1}\left(\Omega^{\prime}\right), \\
f & \mapsto \tilde{u},
\end{aligned}
$$

where $\tilde{u} \in H_{0}^{1}\left(\Omega^{\prime}\right)$ solves $-\nabla^{2} \tilde{u}+\tilde{u}=-\nabla F(\sigma) \cdot \nabla w$ in $\Omega^{\prime}$, and $w \in \tilde{H}^{1}(\Omega)$ is the solution to the adjoint problem

$$
\int_{\Omega} \sigma \nabla w \cdot \nabla v \mathrm{~d} x=\int_{\Gamma} f v \mathrm{~d} s \quad \forall v \in \tilde{H}^{1}(\Omega) .
$$

Proof. For any $\vartheta \in H_{0}^{1}\left(\Omega^{\prime}\right)$, we have by Theorem 2.11

$$
\begin{aligned}
\left\langle f, F^{\prime}(\sigma) \vartheta\right\rangle_{L_{2}(\Gamma)} & =\langle\vartheta,-\nabla F(\sigma) \cdot \nabla w\rangle_{L_{2}\left(\Omega^{\prime}\right)} \\
& =\langle i \vartheta,-\nabla F(\sigma) \cdot \nabla w\rangle_{L_{2}\left(\Omega^{\prime}\right)} \\
& =\left\langle\vartheta, i^{*}(-\nabla F(\sigma) \cdot \nabla w)\right\rangle_{H^{1}\left(\Omega^{\prime}\right)},
\end{aligned}
$$

where $i$ is the embedding operator from $H_{0}^{1}\left(\Omega^{\prime}\right)$ into $L_{2}\left(\Omega^{\prime}\right)$. The assertion follows directly from the expression for the adjoint $i^{*}$ of the embedding operator $i, c f .,[49]$ Corollary 2.3 .

Now we can proceed to the important issue of convergence rate analysis. The estimate bounds the distance between the minimizer $\vartheta_{\alpha}^{\delta}$ and the true solution $\vartheta^{\dagger}$ in terms of the noise level $\delta$ and the parameter $\alpha$, and quantitatively indicates the quality of the approximation. We shall measure the error in Bregman distance [7]. We denote by $\partial R_{r}(\vartheta)$ the subdifferential of the convex functional $R_{r}$ at $\vartheta$, i.e.,

$$
\partial R_{r}(\vartheta)=\left\{\xi \in H_{0}^{1}\left(\Omega^{\prime}\right): R_{r}\left(\vartheta^{\prime}\right)-R_{r}(\vartheta) \geq\left\langle\xi, \vartheta^{\prime}-\vartheta\right\rangle \forall \vartheta^{\prime} \in H_{0}^{1}\left(\Omega^{\prime}\right)\right\},
$$

and define the Bregman distance $d_{\xi}\left(\vartheta, \vartheta^{\dagger}\right)$ between $\vartheta$ and $\vartheta^{\dagger}$ relative to any element $\xi \in \partial R_{r}\left(\vartheta^{\dagger}\right)$ by

$$
d_{\xi}\left(\vartheta, \vartheta^{\dagger}\right)=R_{r}(\vartheta)-R_{r}\left(\vartheta^{\dagger}\right)-\left\langle\xi, \vartheta-\vartheta^{\dagger}\right\rangle .
$$

According to classical regularization theory [20], convergence rates can only be shown under extra conditions on the $R_{r}$-minimizing solution $\vartheta^{\dagger}$. Such conditions are known as source conditions, and usually takes the form of range inclusion. Generally, it is accepted that it represents a certain smoothness requirement. An alternative approach is to use variational inequalities, see [27] for an up-to-date account. Mathematically, the source condition as we shall employ below can be regarded as the necessary optimality condition for the constrained optimization problem ( $c f$. the definition of $\vartheta^{\dagger}$ in (4.2)). We would like to point out that the verification, either theoretical or numerical, of such conditions in general is beyond reach. Readily interpretable source conditions are still under development. Here we shall restrict our attention to the following canonical source condition, and, additionally, sparsity constraint.

Assumption 4.6. Let $\vartheta^{\dagger}$ be an $R_{r}$-minimizing solution.

(a) $\vartheta^{\dagger}$ satisfies the source condition: there exists a $w \in L_{2}(\Gamma)$ such that $\left(F^{\prime}\left(\sigma_{0}\right)\right)^{*} w=\xi \in \partial R_{r}\left(\vartheta^{\dagger}\right)$;

(b) $\vartheta^{\dagger}$ has a finite support $\mathbb{K}$ with respect to $\left\{\psi_{k}\right\}$ and $F^{\prime}\left(\sigma_{0}\right)$ is injective on $\left\{\psi_{k}, k \in \mathbb{K}\right\}$.

Now we can state a first estimate for the linearized model, which follows directly from [8]. 
Theorem 4.7. Let the solution $\vartheta^{\dagger}$ fulfill Assumption 4.6(a). Then for a choice rule $\alpha \sim \delta$, there hold

$$
d_{\xi}\left(\vartheta_{\alpha}^{\delta}, \vartheta^{\dagger}\right) \leq C \delta \quad \text { and } \quad\left\|F^{\prime}\left(\sigma_{0}\right) \vartheta_{\alpha}^{\delta}-\tilde{\phi}^{\delta}\right\|_{L_{2}(\Gamma)} \leq C \delta .
$$

In case of $R_{2}$, Theorem 4.7 immediately gives an estimate in $H^{1}\left(\Omega^{\prime}\right)$-norm. Actually this is true for any $r \in(1,2]$, once noting the following inequality for Bregman distance $d_{\xi}\left(\vartheta, \vartheta^{\dagger}\right)$ [5], Lemma 2.7, [24], Lemma 10.

Lemma 4.8. Let $r \in(1,2]$. There exists a constant $c_{r}>0$ depending only on $r$ such that

$$
d_{\xi}\left(\vartheta^{\prime}, \vartheta\right):=R_{r}\left(\vartheta^{\prime}\right)-R_{r}(\vartheta)-\left\langle\xi, \vartheta^{\prime}-\vartheta\right\rangle \geq \frac{c_{r}\left\|\vartheta^{\prime}-\vartheta\right\|_{H^{1}\left(\Omega^{\prime}\right)}^{2}}{3+2 R_{r}(\vartheta)+R_{r}\left(\vartheta^{\prime}\right)}
$$

for all $\vartheta^{\prime}, \vartheta \in \operatorname{dom}\left(R_{r}\right)$ for which $\partial R_{r}(\vartheta) \neq \emptyset$.

Hence the estimate in Theorem 4.7 implies a convergence rate of $\mathcal{O}\left(\delta^{\frac{1}{2}}\right)$ in the $H^{1}\left(\Omega^{\prime}\right)$-norm. However, the interesting case $R_{1}$ is not covered. This can be remedied by imposing extra conditions $[24,46]$.

Lemma 4.9. Let $r \in[1,2)$ and the solution $\vartheta^{\dagger}$ fulfill Assumption 4.6. Then for the subgradient $\xi \in \partial R_{r}\left(\vartheta^{\dagger}\right)$

$$
\left\|\vartheta-\vartheta^{\dagger}\right\|_{H^{1}\left(\Omega^{\prime}\right)}^{r} \leq C_{1}\left\|F^{\prime}\left(\sigma_{0}\right)\left(\vartheta-\vartheta^{\dagger}\right)\right\|_{L_{2}(\Gamma)}^{r}+C_{2} d_{\xi}\left(\vartheta, \vartheta^{\dagger}\right) .
$$

Proof. The proof can be found in [24], Theorems 14 and 15, for the cases $r>1$ and $r=1$, respectively.

Now we can show an enhanced convergence rate of order $\mathcal{O}\left(\delta^{\frac{1}{r}}\right)$.

Theorem 4.10. Let the conditions in Lemma 4.9 be fulfilled. Then for a choice rule $\alpha \sim \delta$ there holds

$$
\left\|\vartheta_{\alpha}^{\delta}-\vartheta^{\dagger}\right\|_{H^{1}\left(\Omega^{\prime}\right)} \leq C \delta^{\frac{1}{r}}
$$

Proof. By Lemma 4.9, we have

$$
\begin{aligned}
\left\|\vartheta_{\alpha}^{\delta}-\vartheta^{\dagger}\right\|_{H^{1}\left(\Omega^{\prime}\right)}^{r} & \leq C_{1}\left\|F^{\prime}\left(\sigma_{0}\right)\left(\vartheta_{\alpha}^{\delta}-\vartheta^{\dagger}\right)\right\|_{L_{2}(\Gamma)}^{r}+C_{2} d_{\xi}\left(\vartheta_{\alpha}^{\delta}, \vartheta^{\dagger}\right) \\
& \leq C_{1}\left(\left\|F^{\prime}\left(\sigma_{0}\right) \vartheta_{\alpha}^{\delta}-\tilde{\phi}^{\delta}\right\|_{L_{2}(\Gamma)}+\left\|F^{\prime}\left(\sigma_{0}\right) \vartheta^{\dagger}-\tilde{\phi}^{\delta}\right\|_{L_{2}(\Gamma)}\right)^{r}+C_{2} d_{\xi}\left(\vartheta_{\alpha}^{\delta}, \vartheta^{\dagger}\right) .
\end{aligned}
$$

Now the second assertion follows from the choice $\alpha \sim \delta$ and Theorem 4.7.

Remark 4.11. The injectivity of the operator $F^{\prime}\left(\sigma_{0}\right)$ on the finite-dimensional subspace $\left\{\psi_{k}, k \in \mathbb{K}\right\}$ is crucial for deriving the enhanced convergence rate. It holds for piecewise polynomial/analytic conductivity distributions in case that the operator $F(\sigma)$ is the Neumann-to-Dirichlet map [26, 44]. Hence Theorem 4.10 holds with the further restriction on the basis $\left\{\psi_{k}\right\}$ of being piecewise analytic.

Remark 4.12. The current analysis focuses on the a priori choice $\alpha \sim \delta$. However, one can show that under certain conditions, with $\alpha$ chosen by the discrepancy principle, i.e., $\alpha$ satisfies $\left\|F^{\prime}\left(\sigma_{0}\right) \vartheta_{\alpha}^{\delta}-\tilde{\phi}^{\delta}\right\|=c \delta(c \geq 1)$, the solution $\vartheta_{\alpha}^{\delta}$ also converges, and analogous estimates as in Theorems 4.7 and 4.10 hold, see, e.g., [35]. The discrepancy principle is useful if an estimate of the noise level $\delta$ is known. In practice, it is also useful to employ heuristic rules, e.g., balancing principle [34], for which a posteriori estimates can be established. 


\subsection{Nonlinear model}

Now we turn to the full nonlinear model. Some theoretical studies on the nonlinear EIT model have been carried out for the Mumford-Shah and $T V$ penalty in [52] and [51], respectively. Here we consider the $R_{r}$-penalty, which covers both conventional $H^{1}$ - and sparsity penalty, i.e.,

$$
\Psi(\vartheta)=\frac{1}{2}\left\|F(\sigma)-\phi^{\delta}\right\|_{L_{2}(\Gamma)}^{2}+\alpha R_{r}(\vartheta)
$$

where $\vartheta=\sigma-\sigma_{0}$ stands for the inhomogeneity as before. We shall again denote the minimizer to $\Psi$ by $\vartheta_{\alpha}^{\delta}$, and let $\sigma_{\alpha}^{\delta}=\sigma_{0}+\vartheta_{\alpha}^{\delta}$, likewise $\sigma^{\dagger}=\sigma_{0}+\vartheta^{\dagger}$. Under suitable assumptions, such penalties have been treated for general nonlinear operators. We will refer to these results whenever appropriate.

We begin with the following existence, stability and consistency result, i.e., the minimization problem as defined in (4.3) is well-posed.

Theorem 4.13. There exists at least one minimizer $\vartheta_{\alpha}^{\delta}$ to the functional $\Psi(\sigma)$ (over the admissible set $\left.\mathcal{A}\right)$. Let $\left\{\phi^{n}\right\} \subset L_{2}(\Gamma)$ be a sequence of noisy data converging to $\phi^{\delta}$, and $\vartheta^{n}$ be a minimizer to $\Psi$ with $\phi^{n}$ in place of $\phi^{\delta}$. Then the sequence $\left\{\vartheta^{n}\right\}$ has a subsequence converging in $H^{1}\left(\Omega^{\prime}\right)$ to a minimizer of $\Psi$. Moreover, if the parameter $\alpha=\alpha(\delta)$ satisfies $\lim _{\delta \rightarrow 0} \alpha(\delta)=0$ and $\lim _{\delta \rightarrow 0} \frac{\delta^{2}}{\alpha(\delta)}=0$, then the sequence of minimizers $\left\{\vartheta_{\alpha}^{\delta}\right\}$ has a subsequence converging in $H^{1}\left(\Omega^{\prime}\right)$ to an $R_{r}$-minimizing solution $\vartheta^{\dagger}$ as $\delta \rightarrow 0$. Furthermore, if $\vartheta^{\dagger}$ is unique, then the whole sequence converges.

Proof. We only sketch the existence proof. The nonnegativity of $\Psi$ implies the existence of a minimizing sequence $\left\{\vartheta^{n}\right\} \subset \mathcal{A}$, for which $\left\{R_{r}\left(\vartheta^{n}\right)\right\}$ is uniformly bounded. From the inequality $\left\|\vartheta^{n}\right\|_{\ell_{2}} \leq\left\|\vartheta^{n}\right\|_{\ell_{r}} \leq C$ for $r \leq 2$, we deduce the uniform boundedness of $\left\{\vartheta^{n}\right\}$ in $H^{1}\left(\Omega^{\prime}\right)$. Therefore, we can extract a subsequence, also denoted by $\left\{\vartheta^{n}\right\}$, and some $\vartheta^{*} \in H_{0}^{1}(\Omega)$, such that $\vartheta^{n} \rightarrow \vartheta^{*}$ weakly in $H^{1}\left(\Omega^{\prime}\right)$. By Sobolev embedding theorem [21], it converges strongly in $L_{p}\left(\Omega^{\prime}\right)$ for any $p<6$ in case of $d=2,3$. Proposition 2.15 implies $\lim _{n \rightarrow \infty} J\left(\sigma^{n}\right)=J\left(\sigma^{*}\right)$, from which and the weak lower semicontinuity of $R_{r}(\vartheta)$ follows the desired assertion. The rest follows from the general theory of sparsity regularization for nonlinear inverse problems [28].

To obtain convergence rates results, the operator $F(\sigma)$ should be differentiable, and thus Assumption 2.12 is required such that Corollary 2.13 holds. Similar to Section 4.1, we make the following assumptions.

Assumption 4.14. Let $\vartheta^{\dagger}$ be an $R_{r}$-minimizing solution.

(a) $\vartheta^{\dagger}$ satisfies the source condition: there exists a $w \in L_{2}(\Gamma)$ such that $\left(F^{\prime}\left(\sigma^{\dagger}\right)\right)^{*} w=\xi \in \partial R_{r}\left(\vartheta^{\dagger}\right)$ and the norm $\|w\|_{L_{2}(\Gamma)}$ is sufficiently small;

(b) $\vartheta^{\dagger}$ has a finite support $\mathbb{K}$ with respect to $\left\{\psi_{k}\right\}$, and $F^{\prime}\left(\sigma^{\dagger}\right)$ is injective on $\left\{\psi_{k}, k \in \mathbb{K}\right\}$

Next we state a first error estimate for the minimizer $\vartheta_{\alpha}^{\delta}$. The proof is standard [19], but we include it for completeness.

Theorem $4.15(1<r \leq 2)$. Let $r \in(1,2]$ and Assumptions 2.12 and 4.14(a) be fulfilled. Then for a choice rule $\alpha \sim \delta$, there holds

$$
d_{\xi}\left(\vartheta_{\alpha}^{\delta}, \vartheta^{\dagger}\right) \leq C \delta \quad \text { and } \quad\left\|F\left(\sigma_{\alpha}^{\delta}\right)-\phi^{\delta}\right\|_{L_{2}(\Gamma)} \leq C \delta .
$$

Proof. The minimizing property of $\vartheta_{\alpha}^{\delta}$ implies

$$
\frac{1}{2}\left\|F\left(\sigma_{\alpha}^{\delta}\right)-\phi^{\delta}\right\|_{L_{2}(\Gamma)}^{2}+\alpha R_{r}\left(\vartheta_{\alpha}^{\delta}\right) \leq \frac{1}{2}\left\|F\left(\sigma^{\dagger}\right)-\phi^{\delta}\right\|_{L_{2}(\Gamma)}^{2}+\alpha R_{r}\left(\vartheta^{\dagger}\right) .
$$


For a choice rule $\alpha \sim \delta$, this yields $R_{r}\left(\vartheta_{\alpha}^{\delta}\right) \leq C+R_{r}\left(\vartheta^{\dagger}\right)$. Appealing to the source condition in Assumption 4.14(a) and the Cauchy-Schwarz inequality, we arrive at

$$
\begin{aligned}
\frac{1}{2}\left\|F\left(\sigma_{\alpha}^{\delta}\right)-\phi^{\delta}\right\|_{L_{2}(\Gamma)}^{2}+\alpha d_{\xi}\left(\vartheta_{\alpha}^{\delta}, \vartheta^{\dagger}\right) \leq & \frac{1}{2}\left\|F\left(\sigma^{\dagger}\right)-\phi^{\delta}\right\|_{L_{2}(\Gamma)}^{2}-\alpha\left\langle\xi, \vartheta_{\alpha}^{\delta}-\vartheta^{\dagger}\right\rangle \\
\leq & \frac{1}{2}\left\|F\left(\sigma^{\dagger}\right)-\phi^{\delta}\right\|_{L_{2}(\Gamma)}^{2}+\alpha\|w\|_{L_{2}(\Gamma)}\left\|F\left(\sigma_{\alpha}^{\delta}\right)-F\left(\sigma^{\dagger}\right)\right\|_{L_{2}(\Gamma)} \\
& +\alpha\|w\|_{L_{2}(\Gamma)}\left\|\theta\left(\vartheta_{\alpha}^{\delta}, \vartheta^{\dagger}\right)\right\|_{L_{2}(\Gamma)}
\end{aligned}
$$

where the linearization error $\theta\left(\vartheta_{\alpha}^{\delta}, \vartheta^{\dagger}\right)$ is defined as

$$
\theta\left(\vartheta_{\alpha}^{\delta}, \vartheta^{\dagger}\right)=F\left(\sigma_{\alpha}^{\delta}\right)-F\left(\sigma^{\dagger}\right)-F^{\prime}\left(\sigma^{\dagger}\right)\left(\vartheta_{\alpha}^{\delta}-\vartheta^{\dagger}\right)
$$

By the Lipschitz continuity of $F^{\prime}(\sigma)$ in Corollary 2.13, we have

$$
\left\|\theta\left(\vartheta_{\alpha}^{\delta}, \vartheta^{\dagger}\right)\right\|_{L_{2}(\Gamma)} \leq \frac{L}{2}\left\|\vartheta_{\alpha}^{\delta}-\vartheta^{\dagger}\right\|_{H^{1}\left(\Omega^{\prime}\right)}^{2}
$$

With the help of the triangle inequality and Young's inequality, we deduce

$$
\alpha\|w\|_{L_{2}(\Gamma)}\left\|F\left(\sigma_{\alpha}^{\delta}\right)-F\left(\sigma^{\dagger}\right)\right\|_{L_{2}(\Gamma)} \leq \alpha\|w\|_{L_{2}(\Gamma)}\left\|F\left(\sigma^{\dagger}\right)-\phi^{\delta}\right\|_{L_{2}(\Gamma)}+\frac{1}{2} \alpha^{2}\|w\|_{L_{2}(\Gamma)}^{2}+\frac{1}{2}\left\|F\left(\sigma_{\alpha}^{\delta}\right)-\phi^{\delta}\right\|_{L_{2}(\Gamma)}^{2} .
$$

In view of the preceding three inequalities, we arrive at

$$
\alpha\left[d_{\xi}\left(\vartheta_{\alpha}^{\delta}, \vartheta^{\dagger}\right)-\frac{L}{2}\|w\|_{L_{2}(\Gamma)}\left\|\vartheta_{\alpha}^{\delta}-\vartheta^{\dagger}\right\|_{H^{1}\left(\Omega^{\prime}\right)}^{2}\right] \leq \frac{1}{2}\left(\left\|F\left(\sigma^{\dagger}\right)-\phi^{\delta}\right\|_{L_{2}(\Gamma)}+\alpha\|w\|_{L_{2}(\Gamma)}\right)^{2} .
$$

The first assertion follows from this inequality, Lemma 4.8 and the choice of $\alpha$. Next we estimate the term $\left\|F\left(\sigma_{\alpha}^{\delta}\right)-\phi^{\delta}\right\|_{L_{2}(\Gamma)}$. From inequality (4.4), we have

$$
\frac{1}{2}\left\|F\left(\sigma_{\alpha}^{\delta}\right)-\phi^{\delta}\right\|_{L_{2}(\Gamma)}^{2}+\alpha d_{\xi}\left(\vartheta_{\alpha}^{\delta}, \vartheta^{\dagger}\right) \leq \frac{1}{2}\left\|F\left(\sigma^{\dagger}\right)-\phi^{\delta}\right\|_{L_{2}(\Gamma)}^{2}-\alpha\left\langle w, F\left(\sigma_{\alpha}^{\delta}\right)-F\left(\sigma^{\dagger}\right)\right\rangle+\alpha\left\langle w, \theta\left(\vartheta_{\alpha}^{\delta}, \vartheta^{\dagger}\right)\right\rangle,
$$

which upon completing the squares gives

$$
\frac{1}{2}\left\|F\left(\sigma_{\alpha}^{\delta}\right)-\phi^{\delta}+\alpha w\right\|_{L_{2}(\Gamma)}^{2}+\alpha d_{\xi}\left(\vartheta_{\alpha}^{\delta}, \vartheta^{\dagger}\right) \leq \frac{1}{2}\left\|F\left(\sigma^{\dagger}\right)-\phi^{\delta}+\alpha w\right\|_{L_{2}(\Gamma)}^{2}+\alpha\|w\|_{L_{2}(\Gamma)}\left\|\theta\left(\vartheta_{\alpha}^{\delta}, \vartheta^{\dagger}\right)\right\|_{L_{2}(\Gamma)} .
$$

The desired assertion on $\left\|F\left(\sigma_{\alpha}^{\delta}\right)-\phi^{\delta}\right\|_{L_{2}(\Gamma)}$ follows from this and the choice $\alpha \sim \delta$.

Remark 4.16. An inspection of the proof indicates that the restriction on $\|w\|_{L_{2}(\Gamma)}$ depends on three factors: the Lipschitz constant of $F^{\prime}(\sigma)$, the exponent $r$ and $R_{r}\left(\vartheta^{\dagger}\right)$. In case of $r=2$, the Bregman distance $d_{\xi}\left(\vartheta_{\alpha}^{\delta}, \vartheta^{\dagger}\right)$ reduces to $\frac{1}{2}\left\|\vartheta_{\alpha}^{\delta}-\vartheta^{\dagger}\right\|_{H^{1}\left(\Omega^{\prime}\right)}^{2}$, and thus the condition can be explicitly written as $L\|w\|_{L_{2}(\Gamma)}<1[19]$.

By Lemma 4.8, Theorem 4.15 gives a convergence rate $\mathcal{O}\left(\delta^{\frac{1}{2}}\right)$ in $H^{1}\left(\Omega^{\prime}\right)$. The additional condition from Assumption 4.14(b) can enhance the convergence rate from $\mathcal{O}\left(\delta^{\frac{1}{2}}\right)$ to $\mathcal{O}\left(\delta^{\frac{1}{r}}\right)$.

Theorem $4.17(1<r<2)$. Let Assumptions 2.12 and 4.14 be fulfilled. Then for a choice rule $\alpha \sim \delta$ there holds

$$
\left\|\vartheta_{\alpha}^{\delta}-\vartheta^{\dagger}\right\|_{H^{1}\left(\Omega^{\prime}\right)} \leq C \delta^{\frac{1}{r}} .
$$


Proof. By Lemma 4.9, we have

$$
\left\|\vartheta_{\alpha}^{\delta}-\vartheta^{\dagger}\right\|_{H^{1}\left(\Omega^{\prime}\right)}^{r} \leq C_{1}\left\|F^{\prime}\left(\sigma^{\dagger}\right)\left(\vartheta_{\alpha}^{\delta}-\vartheta^{\dagger}\right)\right\|_{L_{2}(\Gamma)}^{r}+C_{2} d_{\xi}\left(\vartheta_{\alpha}^{\delta}, \vartheta^{\dagger}\right) .
$$

We estimate the term $\left\|F^{\prime}\left(\sigma^{\dagger}\right)\left(\vartheta_{\alpha}^{\delta}-\vartheta^{\dagger}\right)\right\|_{L_{2}(\Gamma)}$ by noting (4.5) as follows

$$
\begin{aligned}
\left\|F^{\prime}\left(\sigma^{\dagger}\right)\left(\vartheta_{\alpha}^{\delta}-\vartheta^{\dagger}\right)\right\|_{L_{2}(\Gamma)} & \leq\left\|F\left(\sigma_{\alpha}^{\delta}\right)-F\left(\sigma^{\dagger}\right)\right\|_{L_{2}(\Gamma)}+\left\|F\left(\sigma_{\alpha}^{\delta}\right)-F\left(\sigma^{\dagger}\right)-F^{\prime}\left(\sigma^{\dagger}\right)\left(\vartheta_{\alpha}^{\delta}-\vartheta^{\dagger}\right)\right\|_{L_{2}(\Gamma)} \\
& \leq\left\|F\left(\sigma_{\alpha}^{\delta}\right)-\phi^{\delta}\right\|_{L_{2}(\Gamma)}+\left\|F\left(\sigma^{\dagger}\right)-\phi^{\delta}\right\|_{L_{2}(\Gamma)}+\frac{L}{2}\left\|\vartheta_{\alpha}^{\delta}-\vartheta^{\dagger}\right\|_{H^{1}\left(\Omega^{\prime}\right)}^{2} .
\end{aligned}
$$

The term $\left\|\vartheta_{\alpha}^{\delta}-\vartheta^{\dagger}\right\|_{H^{1}\left(\Omega^{\prime}\right)}$ can be bounded as follows

$$
\left\|\vartheta_{\alpha}^{\delta}-\vartheta^{\dagger}\right\|_{H^{1}\left(\Omega^{\prime}\right)}^{2} \leq C d_{\xi}\left(\vartheta_{\alpha}^{\delta}, \vartheta\right) \leq C \delta
$$

by Lemma 4.8 and Theorem 4.15. The conclusion follows directly from these estimates.

Theorem 4.17 does not cover the case $R_{1}$. Nonetheless, an analogous estimate holds.

Theorem $4.18(r=1)$. Let the solution $\vartheta^{\dagger}$ be unique, and Assumptions 2.12 and 4.14 be fulfilled. Then for small $\delta$ and a choice rule $\alpha \sim \delta$, the following estimate holds

$$
\left\|\vartheta_{\alpha}^{\delta}-\vartheta^{\dagger}\right\|_{H^{1}\left(\Omega^{\prime}\right)} \leq C \delta^{\frac{1}{2}}
$$

Proof. The minimizing property of $\vartheta_{\alpha}^{\delta}$ implies

$$
\frac{1}{2}\left\|F\left(\sigma_{\alpha}^{\delta}\right)-\phi^{\delta}\right\|_{L_{2}(\Gamma)}^{2}+\alpha R_{1}\left(\vartheta_{\alpha}^{\delta}\right) \leq \frac{1}{2}\left\|F\left(\sigma^{\dagger}\right)-\phi^{\delta}\right\|_{L_{2}(\Gamma)}^{2}+\alpha R_{1}\left(\vartheta^{\dagger}\right),
$$

which together with the choice $\alpha \sim \delta$ yields

$$
\left\|F\left(\sigma_{\alpha}^{\delta}\right)-\phi^{\delta}\right\|_{L_{2}(\Gamma)} \leq C_{3} \delta^{\frac{1}{2}} .
$$

Appealing to the source condition $\xi=\left(F^{\prime}\left(\sigma^{\dagger}\right)\right)^{*} w \in \partial R_{1}\left(\vartheta^{\dagger}\right)$ from Assumption 4.14(a) and the definition of the linearization error $\theta\left(\vartheta_{\alpha}^{\delta}, \vartheta^{\dagger}\right)$, i.e., (4.5), we deduce

$$
\begin{aligned}
\frac{1}{2}\left\|F\left(\sigma_{\alpha}^{\delta}\right)-\phi^{\delta}\right\|_{L_{2}(\Gamma)}^{2}+\alpha d_{\xi}\left(\vartheta_{\alpha}^{\delta}, \vartheta^{\dagger}\right) & \leq \frac{1}{2}\left\|F\left(\sigma^{\dagger}\right)-\phi^{\delta}\right\|_{L_{2}(\Gamma)}^{2}-\alpha\left\langle w, F^{\prime}\left(\sigma^{\dagger}\right)\left(\vartheta_{\alpha}^{\delta}-\vartheta^{\dagger}\right)\right\rangle \\
& =\frac{1}{2}\left\|F\left(\sigma^{\dagger}\right)-\phi^{\delta}\right\|_{L_{2}(\Gamma)}^{2}-\alpha\left\langle w, F\left(\sigma_{\alpha}^{\delta}\right)-F\left(\sigma^{\dagger}\right)\right\rangle+\alpha\left\langle w, \theta\left(\vartheta_{\alpha}^{\delta}, \vartheta^{\dagger}\right)\right\rangle .
\end{aligned}
$$

In particular, this together with the choice $\alpha \sim \delta$ implies

$$
d_{\xi}\left(\vartheta_{\alpha}^{\delta}, \vartheta^{\dagger}\right) \leq C_{4} \delta-\left\langle w, F\left(\sigma_{\alpha}^{\delta}\right)-F\left(\sigma^{\dagger}\right)\right\rangle+\left\langle w, \theta\left(\vartheta_{\alpha}^{\delta}, \vartheta^{\dagger}\right)\right\rangle .
$$

Next by Lemma 4.9, we have

$$
\begin{aligned}
\left\|\vartheta_{\alpha}^{\delta}-\vartheta^{\dagger}\right\|_{H^{1}\left(\Omega^{\prime}\right)} & \leq C_{1}\left\|F^{\prime}\left(\sigma^{\dagger}\right)\left(\vartheta_{\alpha}^{\delta}-\vartheta^{\dagger}\right)\right\|_{L_{2}(\Gamma)}+C_{2} d_{\xi}\left(\vartheta_{\alpha}^{\delta}, \vartheta^{\dagger}\right) \\
& \leq C_{1}\left(\left\|F\left(\sigma_{\alpha}^{\delta}\right)-F\left(\sigma^{\dagger}\right)\right\|_{L_{2}(\Gamma)}+\left\|\theta\left(\vartheta_{\alpha}^{\delta}, \vartheta^{\dagger}\right)\right\|_{L_{2}(\Gamma)}\right)+C_{2} d_{\xi}\left(\vartheta_{\alpha}^{\delta}, \vartheta^{\dagger}\right)
\end{aligned}
$$

These two inequalities together with inequality (4.6), the Cauchy-Schwarz inequality and estimate (4.7) yield

$$
\begin{aligned}
\left\|\vartheta_{\alpha}^{\delta}-\vartheta^{\dagger}\right\|_{H^{1}\left(\Omega^{\prime}\right) \leq} & C_{2} C_{4} \delta+\left(C_{1}+C_{2}\|w\|_{L_{2}(\Gamma)}\right)\left\|F\left(\sigma_{\alpha}^{\delta}\right)-F\left(\sigma^{\dagger}\right)\right\|_{L_{2}(\Gamma)} \\
& +\left(C_{1}+C_{2}\|w\|_{L_{2}(\Gamma)}\right)\left\|\theta\left(\vartheta_{\alpha}^{\delta}, \vartheta^{\dagger}\right)\right\|_{L_{2}(\Gamma)} \\
\leq & C_{2} C_{4} \delta+\left(C_{1}+C_{2}\|w\|_{L_{2}(\Gamma)}\right)\left(\delta+C_{3} \delta^{\frac{1}{2}}\right)+\left(C_{1}+C_{2}\|w\|_{L_{2}(\Gamma)}\right) \frac{L}{2}\left\|\vartheta_{\alpha}^{\delta}-\vartheta^{\dagger}\right\|_{H^{1}\left(\Omega^{\prime}\right)}^{2}
\end{aligned}
$$


Upon letting $C_{5}=\left(C_{1}+C_{2}\|w\|_{L_{2}(\Gamma)}\right) \frac{L}{2}$ and $C_{6}=\left(C_{1}+C_{2}\|w\|_{L_{2}(\Gamma)}\right)\left(\delta^{\frac{1}{2}}+C_{3}\right)+C_{2} C_{4} \delta^{\frac{1}{2}}$, this gives $C_{5} t^{2}-$ $t+C_{6} \delta^{\frac{1}{2}} \geq 0$ for $t=\left\|\vartheta_{\alpha}^{\delta}-\vartheta^{\dagger}\right\|_{H^{1}\left(\Omega^{\prime}\right)}$. For sufficiently small $\delta$, we have $1-4 C_{5} C_{6} \delta^{\frac{1}{2}}>0$. Hence, the above quadratic polynomial in $t$ has two distinct positive roots, and the inequality amounts to

$$
t \geq \frac{1+\sqrt{1-4 C_{5} C_{6} \delta^{\frac{1}{2}}}}{2 C_{5}} \text { or } t \leq \frac{1-\sqrt{1-4 C_{5} C_{6} \delta^{\frac{1}{2}}}}{2 C_{5}} .
$$

By virtue of the consistency result in Theorem 4.13, the latter case holds, i.e.,

$$
\left\|\vartheta_{\alpha}^{\delta}-\vartheta^{\dagger}\right\|_{H^{1}\left(\Omega^{\prime}\right)} \leq \frac{1-\sqrt{1-4 C_{5} C_{6} \delta^{\frac{1}{2}}}}{2 C_{5}} \leq 2 C_{6} \delta^{\frac{1}{2}}
$$

where we have utilized the elementary inequality $\sqrt{1-s} \geq 1-s, \forall s \in[0,1]$. This concludes the proof.

Remark 4.19. The estimate (4.7) yields an upper bound for the discrepancy term, which can be improved by a bootstrap argument as follows: the estimate in Theorem 4.18 and applying the Cauchy-Schwarz inequality to (4.8) leads to

$$
\frac{1}{2}\left\|F\left(\sigma_{\alpha}^{\delta}\right)-\phi^{\delta}\right\|_{L_{2}(\Gamma)}^{2} \leq \frac{1}{2} \delta^{2}+\alpha\|w\|_{L_{2}(\Gamma)}\left(\delta+C_{3} \delta^{1 / 2}\right)+\alpha\|w\|_{L_{2}(\Gamma)} \frac{L}{2} \delta
$$

and hence $\left\|F\left(\sigma_{\alpha}^{\delta}\right)-\phi^{\delta}\right\|_{L_{2}(\Gamma)} \leq C \delta^{\frac{3}{4}}$.

This improved estimate for the discrepancy term can then be used to obtain an improved estimate for $\left\|\vartheta_{\alpha}^{\delta}-\vartheta^{\dagger}\right\|_{H^{1}\left(\Omega^{\prime}\right)}$. By repeating the arguments in the proof above we get an estimate of order $\left\|\vartheta_{\alpha}^{\delta}-\vartheta^{\dagger}\right\|_{H^{1}\left(\Omega^{\prime}\right)}=$ $\mathcal{O}\left(\delta^{\frac{3}{4}}\right)$. This bootstrap procedure can be repeated to derive convergence rate of order $\mathcal{O}\left(\delta^{s}\right)$ for any $s<1$.

\section{Concluding Remarks}

In this paper we have presented an analysis of two popular EIT models, i.e., continuum model and complete electrode model, and the continuity and differentiability of the forward operator with respect to $L_{p}$ norms are shown. The analytical results are applied to study several regularization formulations with smoothness/sparsity penalty for the linearized and nonlinear models, which in particular covers the conventional $H^{1}$ penalty and the recent sparsity penalty. The existence of a minimizer, stability and consistency are discussed, and convergence rates are derived. These results partially justify several widely adopted imaging techniques as well as the recent sparsity approach [36], for which excellent empirical performance has been observed previously.

There are several avenues for further research. Firstly, we have restricted our attention to Tikhonov regularization with an $\ell_{r}$-penalty. Alternative approaches, e.g., iterative regularization methods such as Landweber and Gauss-Newton methods in Banach spaces [38], might also be justified using the presented analytical results. Secondly, the operator $F^{\prime}(\sigma)$ deserves further attention, e.g. the solvability of the linearized equation. Thirdly, refined regularity for the forward model is of immense interest. The derivations herein utilize Meyers estimate, which relies only on the $L_{\infty}(\Omega)$ bound of the parameter $\sigma$, and the extra regularity on the conductivity $\sigma, e . g$., $B V$ or $H^{1}$, might enable deriving more refined estimates. Finally, various aspects of numerical implementation of Tikhonov models are of much practical significance, especially in a functional analytic setting. These include an appropriate choice of the functional gradient, verifiable convergence proof of optimization algorithms, e.g., iterative soft shrinkage and Newton type methods, for minimizing relevant Tikhonov models, preconditioning strategies for accelerating convergence, and designing consistent finite-dimensional approximations. The analytical results presented herein are expected to play an essential role in interrogating these important issues.

Acknowledgements. The authors would like to thank two anonymous referees for their constructive comments which have led to an improved presentation of the manuscript. The work of Bangti Jin was substantially supported by the Alexander von Humboldt foundation through a postdoctoral researcher fellowship and is partially supported by Award No. KUS-C1-016-04, made by King Abdullah University of Science and Technology (KAUST), and that of Peter Maass by the German Science Foundation through grant MA 1657/18-1. 


\section{REFERENCES}

[1] G. Alessandrini, Open issues of stability for the inverse conductivity problem. Journal Inverse Ill-Posed Problems 15 (2007) $451-460$.

[2] K. Astala and L. Päivärinta, Calderón's inverse conductivity problem in the plane. Ann. of Math. (2) 163 (2006) $265-299$.

[3] K. Astala, D. Faraco and L. Székelyhidi Jr., Convex integration and the $L^{p}$ theory of elliptic equations. Ann. Scuola Norm. Super. Pisa Cl. Sci. (5) 7 (2008) 1-50.

[4] R.H. Bayford, Bioimpedance tomography (electrical impedance tomography). Ann. Rev. Biomed. Eng. 8 (2006) 63-91.

[5] T. Bonesky, K.S. Kazimierski, P. Maass, F. Schöpfer and T. Schuster, Minimization of Tikhonov functionals in Banach spaces. Abstr. Appl. Anal. (2008) 19 pages.

[6] K. Bredies and D.A. Lorenz, Regularization with non-convex separable constraints. Inverse Problems 25 (2009) 085011.

[7] L.M. Bregman, The relaxation method of finding the common points of convex sets and its application to the solution of problems in convex programming. USSR Comput. Math. Math. Phys. 7 (1967) 200-217.

[8] M. Burger and S. Osher, Convergence rates of convex variational regularization. Inverse Problems 20 (2004) $1411-1420$.

[9] A.-P. Calderón, On an inverse boundary value problem. In Seminar on Numerical Analysis and its Applications to Continuum Physics (Rio de Janeiro, 1980). Soc. Brasil. Mat., Rio de Janeiro (1980) 65-73.

[10] M. Cheney, D. Isaacson, J.C. Newell, S. Simske and J. Goble, NOSER: An algorithm for solving the inverse conductivity problem. Int. J. Imag. Syst. Tech. 2 (1990) 66-75.

[11] M. Cheney, D. Isaacson and J.C. Newell, Electrical impedance tomography. SIAM Rev. 41 (1999) 85-101.

[12] K.-S. Cheng, D. Isaacson, J.C. Newell and D.G. Gisser, Electrode models for electric current computed tomography. IEEE Trans. Biomed. Eng. 36 (1989) 918-924.

[13] E.T. Chung, T.F. Chan and X.-C. Tai, Electrical impedance tomography using level set representation and total variational regularization. J. Comput. Phys. 205 (2005) 357-372.

[14] I. Daubechies, M. Defrise and C. De Mol, An iterative thresholding algorithm for linear inverse problems with a sparsity constraint. Comm. Pure Appl. Math. 57 (2004) 1413-1457.

[15] T. Dierkes, O. Dorn, F. Natterer, V. Palamodov and H. Sielschott, Fréchet derivatives for some bilinear inverse problems. SIAM J. Appl. Math. 62 (2002) 2092-2113.

[16] D. Dobson, Convergence of a reconstruction method for the inverse conductivity problem. SIAM J. Appl. Math. 52 (1992) 442-458.

[17] D.L. Donoho, Compressed sensing. IEEE Trans. Inf. Theor. 52 (2006) 1289-1306.

[18] H. Egger and M. Schlottbom, Analysis and regularization of problems in diffuse optical tomography. SIAM J. Math. Anal. 42 (2010) 1934-1948.

[19] H.W. Engl, K. Kunisch and A. Neubauer, Convergence rates for Tikhonov regularisation of nonlinear ill-posed problems. Inverse Problems 5 (1989) 523-540.

[20] H.W. Engl, M. Hanke and A. Neubauer, Regularization of Inverse Problems. Kluwer Academic, Dordrecht (1996).

[21] L.C. Evans and R.F. Gariepy, Measure Theory and Fine Properties of Functions. CRC Press, Boca Raton (1992).

[22] T. Gallouet and A. Monier, On the regularity of solutions to elliptic equations. Rend. Mat. Appl. (7) 19 (1999) $471-488$.

[23] M. Gehre, T. Kluth, A. Lipponen, B. Jin, A. Seppänen, J. Kaipio and P. Maass, Sparsity reconstruction in electrical impedance tomography: an experimental evaluation. J. Comput. Appl. Math. (2011), in press, DOI: 10.1016/j.cam.2011.09.035.

[24] M. Grasmair, M. Haltmeier and O. Scherzer, Sparse regularization with $l^{q}$ penalty term. Inverse Problems 24 (2008) 055020.

[25] K. Gröger, A $W^{1, p}$-estimate for solutions to mixed boundary value problems for second order elliptic differential equations. Math. Ann. 283 (1989) 679-687.

[26] B. Harrach and J.K. Seo, Exact shape-reconstruction by one-step linearization in electrical impedance tomography. SIAM J. Math. Anal. 42 (2010) 1505-1518.

[27] B. Hofmann and M. Yamamoto, On the interplay of source conditions and variational inequalities for nonlinear ill-posed problems. Appl. Anal. 89 (2010) 1705-1727.

[28] B. Hofmann, B. Kaltenbacher, C. Poeschl and O. Scherzer, A convergence rates result for Tikhonov regularization in Banach spaces with non-smooth operators. Inverse Problems 23 (2007) 987-1010.

[29] N. Hyvönen, Complete electrode model of electrical impedance tomography: approximation properties and characterization of inclusions. SIAM J. Appl. Math. 64 (2004) 902-931.

[30] M. Ikehata and S. Siltanen, Electrical impedance tomography and Mittag-Leffler's function. Inverse Problems 20 (2004) 1325-1348.

[31] O.Y. Imanuvilov, G. Uhlmann and M. Yamamoto, The Calderón problem with partial data in two dimensions. J. Amer. Math. Soc. 23 (2010) 655-691.

[32] D. Isaacson, J.L. Mueller, J.C. Newell and S. Siltanen, Reconstructions of chest phantoms by the D-bar method for electrical impedance tomography. IEEE Trans. Med. Imag. 23 (2004) 821-828.

[33] K. Ito, K. Kunisch and Z. Li, Level-set function approach to an inverse interface problem. Inverse Problems 17 (2001) $1225-1242$.

[34] K. Ito, B. Jin and T. Takeuchi, A regularization parameter for nonsmooth Tikhonov regularization. SIAM J. Sci. Comput. 33 (2011) 1415-1438.

[35] B. Jin, Y. Zhao and J. Zou, Iterative parameter choice by discrepancy principle. IMA J. Numer. Anal. (2011), in press. 
[36] B. Jin, Y. Zhao and P. Maass, A reconstruction algorithm for electrical impedance tomography based on sparsity regularization. Internat. J. Numer. Methods Engrg. (2011), DOI: 10.2002/nme.3247.

[37] J.P. Kaipio, V. Kolehmainen, E. Somersalo and M. Vauhkonen, Statistical inversion and Monte Carlo sampling methods in electrical impedance tomography. Inverse Problems 16 (2000) 1487-1522.

[38] B. Kaltenbacher and B. Hofmann, Convergence rates for the iteratively regularized Gauss-Newton method in Banach spaces. Inverse Problems 26 (2010) 035007.

[39] A. Kirsch and N. Grinberg, The Factorization Method for Inverse Problems. Oxford University Press, Oxford (2008).

[40] K. Knudsen, M. Lassas, J.L. Mueller and S. Siltanen, Regularized D-bar method for the inverse conductivity problem. IPI 3 (2009) 599-624.

[41] V. Kolehmain, A. Voutilainen and J.P. Kaipio, Estimation of nonstionary region boundaries in EIT-state estimation approach. Inverse Problems 17 (2001) 1937-1956.

[42] A. Lechleiter, A regularization technique for the factorization method. Inverse Problems 22 (2006) $1605-1625$.

[43] A. Lechleiter and A. Rieder, Newton regularizations for impedance tomography: a numerical study. Inverse Problems 22 (2006) 1967-1987.

[44] A. Lechleiter and A. Rieder, Newton regularizations for impedance tomography: convergence by local injectivity. Inverse Problems, 24 (2008) 065009.

[45] W.R.B. Lionheart, EIT reconstruction algorithms: pitfalls, challenges and recent developments. Physiol. Meas. 25 (2004) $125-142$.

[46] D.A. Lorenz, Convergence rates and source conditions for Tikhonov regularization with sparsity constraints. Journal Inverse Ill-Posed Problems 16 (2008) 463-478.

[47] M. Lukaschewitsch, P. Maass and M. Pidcock, Tikhonov regularization for electrical impedance tomography on unbounded domains. Inverse Problems 19 (2003) 585-610.

[48] N.G. Meyers, An $L^{p}$-estimate for the gradient of solutions of second order elliptic divergence equations. Ann. Scuola Norm. Sup. Pisa (3) 17 (1963) 189-206.

[49] A. Neubauer, When do Sobolev spaces form a Hilbert scale? Proc. Amer. Math. Soc. 103 (1988) 557-562.

[50] E. Resmerita, Regularization of ill-posed problems in Banach spaces: convergence rates. Inverse Problems 21 (2005) $1303-1314$.

[51] L. Rondi, On the regularization of the inverse conductivity problem with discontinuous conductivities. IPI 2 (2008) 397-409.

[52] L. Rondi and F. Santosa, Enhanced electrical impedance tomography via the Mumford-Shah functional. ESAIM Control Optim. Calc. Var. 6 (2001) 517-538.

[53] E. Somersalo, M. Cheney and D. Isaacson, Existence and uniqueness for electrode models for electric current computed tomography. SIAM J. Appl. Math. 52 (1992) 1023-1040.

[54] A.N. Tikhonov and V.Y. Arsenin, Solutions of Ill-Posed Problems. John Wiley, New York (1977).

[55] G. Uhlmann, Commentary on Calderón's paper (29), on an inverse boundary value problem, in Selected papers of Alberto P. Calderón. Amer. Math. Soc., Providence, RI (2008) 623-636.

[56] A. Wexler, B. Fry and M.R. Neuman, Impedance-computed tomography algorithm and system. Appl. Opt. 24 (1985) 39853992.

[57] T.J. Yorkey, J.G. Webster and W.J. Tompkins, Comparing reconstruction algorithms for electrical impedance tomography. IEEE Trans. Biomed. Eng. 34 (1987) 843-852. 